\title{
Crossed modules for Lie 2-algebras
}

\author{
Honglei Lang and Zhangju Liu \\ Department of Mathematics and LMAM \\ Peking University, Beijing 100871, China \\ email: hllang@pku.edu.cn; liuzj@pku.edu.cn
}

\begin{abstract}
The notion of crossed modules for Lie 2-algebras is introduced. We show that, associated to such a crossed module, there is a strict Lie 3-algebra structure on its mapping cone complex and a strict Lie 2-algebra structure on its derivations. Finally, we classify strong crossed modules by means of the third cohomology group of Lie 2-algebras.
\end{abstract}

\section{Contents}

1 Introduction 1

2 Background on Lie 2-algebras 2

2.1 Basic notions . . . . . . . . . . . . . . . . . . . . . . . 2

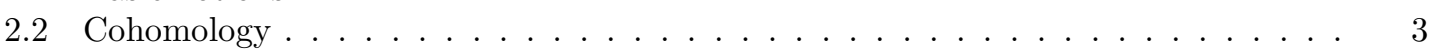

2.3 Derivations . . . . . . . . . . . . . . . . . . . . . . . .

3 Crossed modules of Lie 2-algebras 6

3.1 Definition of crossed modules . . . . . . . . . . . . . . . . . . 6

3.2 Lie 3 -algebras associated to crossed modules . . . . . . . . . . . . . . . . 9

3.3 Examples ............................. 10

4 The first cohomology and derivations of crossed modules

4.1 Lie algebra structures on $C^{1}(\mathfrak{g}, \mathbb{V}) \ldots \ldots \ldots \ldots \ldots$

4.2 Derivations of crossed modules . . . . . . . . . . . . . . . . . . 13

5 Classification of strong crossed modules via $\mathrm{H}^{3} \quad \mathbf{1 6}$

5.1 Crossed modules from extensions of short exact sequences . . . . . . . . . . . . 16

5.2 Classification . . . . . . . . . . . . . . . . . . . . . . 19

\section{Introduction}

Crossed modules of Lie algebras first appeared in the work of Gerstenhaber (11), which can be classified by use of the third cohomology group of Lie algebras as follows: for a crossed module of Lie algebras $\varphi: \mathfrak{m} \longrightarrow \mathfrak{g}$, there exists a four term exact sequence

$$
0 \longrightarrow \mathbb{V} \stackrel{i}{\longrightarrow} \mathfrak{m} \stackrel{\varphi}{\longrightarrow} \mathfrak{g} \stackrel{\pi}{\longrightarrow} \mathfrak{h} \longrightarrow 0,
$$

\footnotetext{
${ }^{0}$ Keyword: crossed module, Lie 2-algebra, derivations.
} 
where the cokernel $\mathfrak{h}$ is a Lie algebra and the kernel $\mathbb{V}$ is an $\mathfrak{h}$-module induced by the action of $\mathfrak{g}$ on $\mathfrak{m}$. Denote by $\operatorname{crmod}(\mathfrak{h}, \mathbb{V})$ the set of equivalence classes of crossed modules with fixed kernel $\mathbb{V}$, cokernel $\mathfrak{h}$ and action. Gerstenhaber proved that there is a bijection between $\operatorname{crmod}(\mathfrak{h}, \mathbb{V})$ and $\mathrm{H}^{3}(\mathfrak{h}, \mathbb{V})$. See also 24] for more details and [3, 4, for other algebraic structures.

Lie algebras can be categorified to Lie 2-algebras. For a good introduction on this subject see 1, 14, 6. A Lie 2-algebra is a 2-vector space equipped with a skew-symmetric bilinear functor, such that the Jacobi identity is controlled by a natural isomorphism, which satisfies the coherence law of its own. It is well-known that the notion of Lie 2-algebras is equivalent to that of 2-term $L_{\infty}$-algebras and the category of strict Lie 2-algebras is isomorphic to the category of crossed modules of Lie algebras ([1]).

The cohomology of $L_{\infty}$-algebras and $A_{\infty}$-algebras was studied in [14, 22, 16] and in [18, for a more general theory. While the cohomology of Lie 2-algebras was formulated in 2 for the strict case to characterize strict Lie 2-bialgebras and in [15] for the general case to depict the deformation of Lie 2-algebras. For the cohomology of Lie 2-groups, see 12. In this paper, we propose the notion of crossed modules of Lie 2-algebras (Definition 3.2) and classify strong crossed modules via the third cohomology group of Lie 2-algebras (Theorem 5.10).

Moreover, given a crossed module of Lie 2-algebras $(\mathfrak{m}, \mathfrak{g}, \phi, \varphi, \sigma)$, there is a strict Lie 3 -algebra $\left(l_{4}=0\right)$ structure on its mapping cone complex: $\mathfrak{m}_{1} \longrightarrow \mathfrak{g}_{1} \oplus \mathfrak{m}_{0} \longrightarrow \mathfrak{g}_{0}$ (Theorem 3.5). Also, we obtain a strict Lie 2-algebra structure on derivations $\operatorname{Der}(\mathfrak{g}, \mathfrak{m}): \operatorname{Hom}\left(\mathfrak{g}_{0}, \mathfrak{m}_{1}\right) \stackrel{-\mathrm{D}}{\longrightarrow} \operatorname{Der}_{0}(\mathfrak{g}, \mathfrak{m})$ (Theorem 4.4), where $\operatorname{Der}_{0}(\mathfrak{g}, \mathfrak{m})$ is the set of 1-cocycles and D is the Lie 2-algebra coboundary operator. Moreover, we get a Lie algebra structure on the first cohomology group $\mathrm{H}^{1}(\mathfrak{g}, \mathfrak{m})$.

This paper is organized as follows: In Section 2, we sketch some background on Lie 2-algebras, including basic definitions, the cohomology theory and the derivations of Lie 2-algebras. In Section 3. we introduce the notion of crossed modules of Lie 2-algebras with some examples and demonstrate that there is a strict Lie 3-algebra on the mapping cone complex. In Section 4 , we provide a Lie algebra structure on the set of 1-cocycles. Then we prove that for a crossed module, there exists a strict Lie 2-algebra structure on its derivations $\operatorname{Der}(\mathfrak{g}, \mathfrak{m})$ and a Lie algebra structure on $\mathrm{H}^{1}(\mathfrak{g}, \mathfrak{m})$. Section 5 is concerned about the classification of strong crossed modules using the third cohomology group.

Acknowledgement: We would like to thank M. Markl for his useful comments on free Lie 2algebras.

\section{Background on Lie 2-algebras}

\subsection{Basic notions}

$L_{\infty}$-algebras, also called strongly homotopy Lie algebras, were introduced by Drinfeld and Stasheff as a model for "Lie algebras that satisfy Jacobi identity up to all higher homotopies". The following definition of $L_{\infty}$-structure was formulated by Stasheff in 1985. See [23].

Definition 2.1. An $L_{\infty}$-algebra is a graded vector space $\mathfrak{g}=\mathfrak{g}_{0} \oplus \mathfrak{g}_{1} \oplus \cdots$ equipped with a system $\left\{l_{k} \mid 1 \leq k<\infty\right\}$ of linear maps $l_{k}: \wedge^{k} \mathfrak{g} \longrightarrow \mathfrak{g}$ with degree $\operatorname{deg}\left(l_{k}\right)=k-2$, where the exterior powers are interpreted in the graded sense and the following relation with Koszul sign "Ksgn" is satisfied for all $n \geq 0$ :

$$
\sum_{i+j=n+1}(-1)^{i(j-1)} \sum_{\sigma} \operatorname{sgn}(\sigma) K \operatorname{sgn}(\sigma) l_{j}\left(l_{i}\left(x_{\sigma(1)}, \cdots, x_{\sigma(i)}\right), x_{\sigma(i+1)}, \cdots, x_{\sigma(n)}\right)=0,
$$

where the summation is taken over all $(i, n-i)$-unshuffles with $i \geq 1$. 
Usually, an $n$-term $L_{\infty}$-algebra (i.e., $l_{i}=0, i \geq n+2$ ) is called a Lie $n$-algebra. In particular, if $l_{n+1}=0$, it is called a strict Lie $n$-algebra. Next we focus on the case of $n=2$.

Definition 2.2. Let $\left(\mathfrak{g}, d, l_{2}, l_{3}\right)$ and $\left(\mathfrak{g}^{\prime}, d^{\prime}, l_{2}^{\prime}, l_{3}^{\prime}\right)$ be Lie 2-algebras. A Lie 2-algebra homomorphism $\varphi: \mathfrak{g} \longrightarrow \mathfrak{g}^{\prime}$ consists of

- two linear maps $\varphi_{0}: \mathfrak{g}_{0} \longrightarrow \mathfrak{g}_{0}^{\prime}$ and $\varphi_{1}: \mathfrak{g}_{1} \longrightarrow \mathfrak{g}_{1}^{\prime}$,

- one bilinear map $\varphi_{2}: \mathfrak{g}_{0} \wedge \mathfrak{g}_{0} \longrightarrow \mathfrak{g}_{1}^{\prime}$,

such that the following equalities hold for all $x, y, z \in \mathfrak{g}_{0}, a \in \mathfrak{g}_{1}$ :

- $d^{\prime} \circ \varphi_{1}=\varphi_{0} \circ d$,

- $\varphi_{0} l_{2}(x, y)-l_{2}^{\prime}\left(\varphi_{0}(x), \varphi_{0}(y)\right)=d^{\prime} \varphi_{2}(x, y)$,

- $\varphi_{1} l_{2}(x, a)-l_{2}^{\prime}\left(\varphi_{0}(x), \varphi_{1}(a)\right)=\varphi_{2}(x, d a)$,

- $l_{2}^{\prime}\left(\varphi_{0}(x), \varphi_{2}(y, z)\right)+$ c.p. $+l_{3}^{\prime}\left(\varphi_{0}(x), \varphi_{0}(y), \varphi_{0}(z)\right)=\varphi_{2}\left(l_{2}(x, y), z\right)+c . p .+\varphi_{1}\left(l_{3}(x, y, z)\right)$,

where c.p. means cyclic permutation. It is called a strong homomorphism if $\varphi_{2}=0$.

Lemma 2.3. Let $\left(\mathfrak{g}, d, l_{2}, l_{3}\right)$ be a Lie 2-algebra and $\mathfrak{h} \subset \mathfrak{g}$ a 2-vector subspace. Then $\mathfrak{g} / \mathfrak{h}$ is a quotient Lie 2-algebra if and only if

$$
l_{2}(\mathfrak{h} \wedge \mathfrak{g}) \subset \mathfrak{h}, \quad l_{3}\left(\mathfrak{h}_{0} \wedge \mathfrak{g}_{0} \wedge \mathfrak{g}_{0}\right) \subset \mathfrak{h}_{1} .
$$

We call $\mathfrak{h}$ satisfying condition (2) an ideal of $\mathfrak{g}$. In fact, the projection $\pi: \mathfrak{g} \longrightarrow \mathfrak{g} / \mathfrak{h}$ becomes a strong homomorphism. We now give an analogue of the fundamental theorem of algebras for Lie 2-algebras.

Proposition 2.4. Let $\varphi: \mathfrak{g} \longrightarrow \mathfrak{g}^{\prime}$ be a Lie 2-algebra homomorphism. Then,

(1) $\operatorname{Im} \varphi=\operatorname{Im} \varphi_{0} \oplus \operatorname{Im} \varphi_{1}$ is a Lie 2-subalgebra of $\mathfrak{g}^{\prime}$ if $\operatorname{Im} \varphi_{2} \subset \operatorname{Im} \varphi_{1}$;

(2) $\operatorname{ker} \varphi=\operatorname{ker} \varphi_{0} \oplus \operatorname{ker} \varphi_{1}$ is an ideal of $\mathfrak{g}$ if $\varphi_{2}\left(\operatorname{ker} \varphi_{0} \wedge \mathfrak{g}_{0}\right)=0$.

Moreover, the two Lie 2-algebras $\mathfrak{g} / \operatorname{ker} \varphi$ and $\operatorname{Im} \varphi$ are isomorphic if the two conditions above are satisfied.

Proof. By Definition 2.2 and $\operatorname{Im} \varphi_{2} \subset \operatorname{Im} \varphi_{1}$, it is direct to see that $\operatorname{Im} \varphi$ is a 2-vector subspace of $\mathfrak{g}^{\prime}$ such that $l_{2}^{\prime}, l_{3}^{\prime}$ are closed on it. Namely, $\operatorname{Im} \varphi$ is a Lie 2 -subalgebra of $\mathfrak{g}^{\prime}$.

Similarly, by the first three conditions of a Lie 2-algebra homomorphism and $\varphi_{2}\left(\operatorname{ker} \varphi_{0} \wedge \mathfrak{g}_{0}\right)=0$, we get $l_{2}\left(\operatorname{ker} \varphi_{0} \wedge \mathfrak{g}\right) \subset \operatorname{ker} \varphi$. Coupled with the last condition, we further obtain that $l_{3}\left(\operatorname{ker} \varphi_{0} \wedge\right.$ $\left.\mathfrak{g}_{0} \wedge \mathfrak{g}_{0}\right) \subset \operatorname{ker} \varphi_{1}$. That is, $\operatorname{ker} \varphi$ is an ideal of $\mathfrak{g}$. The remaining result is immediate.

\subsection{Cohomology}

Given a $\mathfrak{g}$-module $\mathbb{V}: V_{1} \stackrel{\partial}{\longrightarrow} V_{0}$ with a Lie 2-algebra homomorphism $\phi: \mathfrak{g} \rightarrow \operatorname{End}(\mathbb{V}), \phi$ is called an action of $\mathfrak{g}$ on $\mathbb{V}$ and denoted by:

$$
x \triangleright u=\phi(x) u, \quad(x, y) \triangleright u=\phi_{2}(x, y) u, \forall x, y \in \mathfrak{g}, u \in \mathbb{V} .
$$


The cohomology group of a Lie 2 -algebra $\left(\mathfrak{g}, d, l_{2}, l_{3}\right)$ comes from the generalized Chevalley-Eilenberg complex as follows:

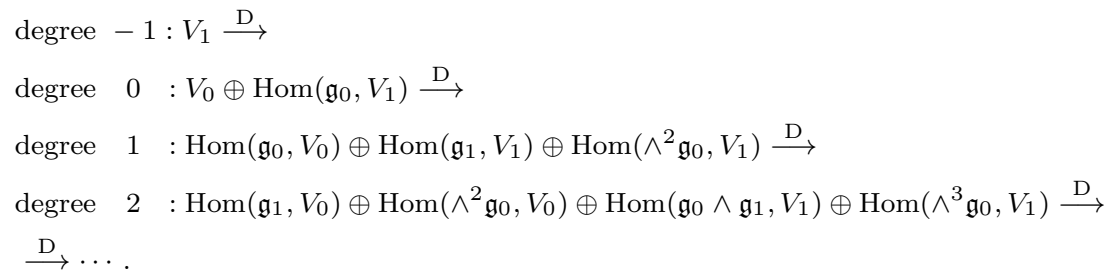

Denote by $C^{i}(\mathfrak{g}, \mathbb{V})$ the set of i-cochains. The coboundary operator $\mathrm{D}$ can be decomposed as:

$$
\mathrm{D}=\hat{d}+\hat{\partial}+d_{\phi}^{(1,0)}+d_{\phi}^{(0,1)}+d_{\phi_{2}}+d_{l_{3}},
$$

where, for $s=0,1$,

$$
\begin{aligned}
& \left.\hat{d}: \operatorname{Hom}\left(\wedge^{p} \mathfrak{g}_{0} \wedge \odot^{q} \mathfrak{g}_{1}, V_{s}\right) \longrightarrow \operatorname{Hom}\left(\wedge^{p-1} \mathfrak{g}_{0} \wedge \odot^{q+1} \mathfrak{g}_{1}\right), V_{s}\right), \\
& \hat{\partial}: \operatorname{Hom}\left(\wedge^{p} \mathfrak{g}_{0} \wedge \odot^{q} \mathfrak{g}_{1}, V_{1}\right) \longrightarrow \operatorname{Hom}\left(\wedge^{p} \mathfrak{g}_{0} \wedge \odot{ }^{q} \mathfrak{g}_{1}, V_{0}\right), \\
& d_{\phi}^{(1,0)}: \operatorname{Hom}\left(\wedge^{p} \mathfrak{g}_{0} \wedge \odot^{q} \mathfrak{g}_{1}, V_{s}\right) \longrightarrow \operatorname{Hom}\left(\wedge^{p+1} \mathfrak{g}_{0} \wedge \odot^{q} \mathfrak{g}_{1}, V_{s}\right), \\
& d_{\phi}^{(0,1)}: \operatorname{Hom}\left(\wedge^{p} \mathfrak{g}_{0} \wedge \odot^{q} \mathfrak{g}_{1}, V_{0}\right) \quad \longrightarrow \quad \operatorname{Hom}\left(\wedge^{p} \mathfrak{g}_{0} \wedge \odot^{q+1} \mathfrak{g}_{1}, V_{1}\right), \\
& d_{\phi_{2}}: \operatorname{Hom}\left(\wedge^{p} \mathfrak{g}_{0} \wedge \odot^{q} \mathfrak{g}_{1}, V_{0}\right) \quad \longrightarrow \operatorname{Hom}\left(\wedge^{p+2} \mathfrak{g}_{0} \wedge \odot^{q} \mathfrak{g}_{1}, V_{1}\right), \\
& d_{l_{3}}: \operatorname{Hom}\left(\wedge^{p} \mathfrak{g}_{0} \wedge \odot^{q} \mathfrak{g}_{1}, V_{s}\right) \quad \longrightarrow \quad \operatorname{Hom}\left(\wedge^{p+3} \mathfrak{g}_{0} \wedge \odot^{q-1} \mathfrak{g}_{1}, V_{s}\right) .
\end{aligned}
$$

More concretely, for any $x_{i} \in \mathfrak{g}_{0}, a_{i} \in \mathfrak{g}_{1}, i \in \mathbb{N}$,

$$
\begin{aligned}
& \hat{d} f\left(x_{1}, \cdots, x_{p-1}, a_{1}, \cdots, a_{q+1}\right)=(-1)^{p}\left(f\left(x_{1}, \cdots, x_{p-1}, d h_{1}, h_{2}, \cdots, h_{q+1}\right)+c . p \cdot\left(a_{1}, \cdots, a_{q+1}\right)\right), \\
& \hat{\partial} f=(-1)^{p+2 q} \partial \circ f, \\
& d_{\phi}^{(1,0)} f\left(x_{1}, \cdots, x_{p+1}, a_{1}, \cdots, a_{q}\right)=\sum_{i=1}^{p+1}(-1)^{i+1} x_{i} \triangleright f\left(x_{1}, \cdots, \widehat{x_{i}}, \cdots, x_{p+1}, a_{1}, \cdots, a_{q}\right) \\
& +\sum_{i<j}(-1)^{i+j} f\left(\left[x_{i}, x_{j}\right], x_{1}, \cdots, \widehat{x_{i}}, \cdots, \widehat{x_{j}}, \cdots, x_{p+1}, a_{1}, \cdots, a_{q}\right) \\
& +\sum_{i, j}(-1)^{i} f\left(x_{1}, \cdots, \widehat{x_{i}}, \cdots, x_{p+1}, a_{1}, \cdots,\left[x_{i}, a_{j}\right], \cdots, a_{q}\right) \\
& d_{\phi}^{(0,1)} f\left(x_{1}, \cdots, x_{p}, a_{1}, \cdots, a_{q+1}\right)=\sum_{i=1}^{q+1}(-1)^{p} a_{i} \triangleright f\left(x_{1}, \cdots, x_{p}, a_{1}, \cdots, \widehat{a_{i}}, \cdots, a_{q+1}\right), \\
& d_{\phi_{2}} f\left(x_{1}, \cdots, x_{p+2}, a_{1}, \cdots, a_{q}\right)=\sum_{\sigma}(-1)^{p+2 q}(-1)^{\sigma}\left(x_{\sigma(1)}, x_{\sigma(2)}\right) \triangleright f\left(x_{\sigma(3)}, \cdots, x_{\sigma(p+2)}, a_{1}, \cdots, a_{q}\right), \\
& d_{l_{3}} f\left(x_{1}, \cdots, x_{p+3}, a_{1}, \cdots, a_{q-1}\right)=\sum_{\tau}-(-1)^{\tau} f\left(x_{\tau(4)}, \cdots, x_{\tau(p+3)}, a_{1}, \cdots, a_{q-1}, l_{3}\left(x_{\tau(1)}, x_{\tau(2)}, x_{\tau(3)}\right)\right) .
\end{aligned}
$$

where $\sigma$ and $\tau$ are taken over all $(2, p)$-unshuffles and $(3, p)$-unshuffles respectively.

By direct calculations, we get the expressions for 1-cocycles and 1-coboundaries.

Lemma 2.5. Let $X+l_{X} \in C^{1}(\mathfrak{g}, \mathbb{V})$, where $X=\left(X_{0}, X_{1}\right) \in \operatorname{Hom}\left(\mathfrak{g}_{0}, V_{0}\right) \oplus \operatorname{Hom}\left(\mathfrak{g}_{1}, V_{1}\right)$ and $l_{X} \in \operatorname{Hom}\left(\wedge^{2} \mathfrak{g}_{0}, V_{1}\right)$. Then

(1) $\mathrm{D}\left(X+l_{X}\right)=0$ if and only if

$$
\begin{aligned}
X_{0} \circ d & =\partial \circ X_{1}, \\
\partial l_{X}(x, y) & =X[x, y]+y \triangleright X x-x \triangleright X y, \\
l_{X}(x, d a) & =X[x, a]+a \triangleright X x-x \triangleright X a, \\
X l_{3}(x, y, z) & =l_{X}(x,[y, z])+x \triangleright l_{X}(y, z)-(y, z) \triangleright X x+c . p .(x, y, z) .
\end{aligned}
$$


(2) $\exists u+\Theta \in V_{0} \oplus \operatorname{Hom}\left(\mathfrak{g}_{0}, V_{1}\right)=C^{0}(\mathfrak{g}, \mathbb{V})$, s.t. $X+l_{X}=\mathrm{D}(u+\Theta)$ if and only if

$$
\begin{aligned}
X(x+a) & =x \triangleright u+a \triangleright u-\partial \Theta(x)-\Theta(d a), \\
l_{X}(x, y) & =(x, y) \triangleright u+x \triangleright \Theta(y)-y \triangleright \Theta(x)-\Theta([x, y]) .
\end{aligned}
$$

For future references, we specify the 3 -coboundaries. For a cochain $\lambda=\sum_{i=0}^{3} \lambda_{i} \in C^{2}(\mathfrak{g}, \mathbb{V})$, where

$$
\lambda_{0} \in \operatorname{Hom}\left(\mathfrak{g}_{1}, V_{0}\right), \lambda_{1} \in \operatorname{Hom}\left(\wedge^{2} \mathfrak{g}_{0}, V_{0}\right), \lambda_{2} \in \operatorname{Hom}\left(\mathfrak{g}_{0} \wedge \mathfrak{g}_{1}, V_{1}\right), \lambda_{3} \in \operatorname{Hom}\left(\wedge^{3} \mathfrak{g}_{0}, V_{1}\right),
$$

then $\theta=\mathrm{D} \lambda$ has five components as follows:

$$
\left\{\begin{array}{rlrl}
\theta_{0} & =d_{\phi}^{(1,0)} \lambda_{0}+\hat{d} \lambda_{1}+\hat{\partial} \lambda_{2} & & \in \operatorname{Hom}\left(\mathfrak{g}_{0} \wedge \mathfrak{g}_{1}, V_{0}\right), \\
\theta_{1} & =d_{\phi}^{(0,1)} \lambda_{0}+\hat{d} \lambda_{2} & & \in \operatorname{Hom}\left(\odot^{2} \mathfrak{g}_{1}, V_{1}\right), \\
\theta_{2} & =d_{l_{3}} \lambda_{0}+d_{\phi}^{(1,0)} \lambda_{1}+\hat{\partial} \lambda_{3} & & \in \operatorname{Hom}\left(\wedge^{3} \mathfrak{g}_{0}, V_{0}\right), \\
\theta_{3} & =d_{\phi_{2}} \lambda_{0}+d_{\phi}^{(0,1)} \lambda_{1}+d_{\phi}^{(1,0)} \lambda_{2}+\hat{d} \lambda_{3} & & \in \operatorname{Hom}\left(\wedge^{2} \mathfrak{g}_{0} \wedge \mathfrak{g}_{1}, V_{1}\right), \\
\theta_{4}=d_{\phi_{2}} \lambda_{1}+d_{l_{3}} \lambda_{2}+d_{\phi}^{(1,0)} \lambda_{3} & & \in \operatorname{Hom}\left(\wedge^{4} \mathfrak{g}_{0}, V_{1}\right) .
\end{array}\right.
$$

More precisely, for any $x, y, z, x_{i} \in \mathfrak{g}_{0}, a, b \in \mathfrak{g}_{1}$,

$$
\left\{\begin{aligned}
\theta_{0}(x, a)= & x \triangleright \lambda_{0}(a)-\lambda_{0}[x, a]+\lambda_{1}(x, d a)-\partial \lambda_{2}(x, a), \\
\theta_{1}(a, b)= & a \triangleright \lambda_{0}(b)+b \triangleright \lambda_{0}(a)-\lambda_{2}(d a, b)-\lambda_{2}(d b, a), \\
\theta_{2}(x, y, z)= & -\lambda_{0} l_{3}(x, y, z)+\left(x \triangleright \lambda_{1}(y, z)-\lambda_{1}([x, y], z)+c . p .\right)-\partial \lambda_{3}(x, y, z), \\
\theta_{3}(x, y, a)= & (x, y) \triangleright \lambda_{0}(a)+a \triangleright \lambda_{1}(x, y)+x \triangleright \lambda_{2}(y, u)-y \triangleright \lambda_{2}(x, a)-\lambda_{2}([x, y], a) \\
& -\lambda_{2}(y,[x, a])+\lambda_{2}(x,[y, a])-\lambda_{3}(x, y, d a), \\
\theta_{4}\left(x_{1}, \cdots, x_{4}\right)= & \sum_{\sigma}(-1)^{\sigma}\left(x_{\sigma_{1}}, x_{\sigma_{2}}\right) \triangleright \lambda_{1}\left(x_{\sigma_{3}}, x_{\sigma_{4}}\right)-\sum_{\tau}(-1)^{\tau} \lambda_{2}\left(x_{\tau_{4}}, l_{3}\left(x_{\tau_{1}}, x_{\tau_{2}}, x_{\tau_{3}}\right)\right) \\
& +\sum_{i=1}^{4}(-1)^{i+1} x_{i} \triangleright \lambda_{3}\left(x_{1}, \cdots, \widehat{x_{i}}, \cdots, x_{4}\right) \\
& +\sum_{i<j}(-1)^{i+j} \lambda_{3}\left(\left[x_{i}, x_{j}\right], x_{1}, \cdots, \widehat{x_{i}}, \cdots, \widehat{x_{j}}, \cdots, x_{4}\right) .
\end{aligned}\right.
$$

\subsection{Derivations}

For a Lie 2-algebra $\left(\mathfrak{g}, d,[\cdot, \cdot], l_{3}\right)$, there is a natural adjoint action ad of $\mathfrak{g}$ on itself given by

$$
a d(x)=[x, \cdot], \quad a d_{2}(y, z)=-l_{3}(y, z, \cdot), \quad \forall x, y, z \in \mathfrak{g} .
$$

To propose the crossed module of Lie 2-algebras below, we review the notion of derivations of a Lie 2-algebra $\operatorname{Der}(\mathfrak{g})$, which was proved to be a strict Lie 2-algebra in [5].

Let $\left(\mathfrak{g}, d,[\cdot, \cdot], l_{3}\right)$ be a Lie 2-algebra. A derivation of degree 0 of $\mathfrak{g}$ is a pair $\left(X, l_{X}\right)$, also denoted by $X+l_{X}$, where $X=\left(X_{0}, X_{1}\right) \in \operatorname{Hom}\left(\mathfrak{g}_{0}, \mathfrak{g}_{0}\right) \oplus \operatorname{Hom}\left(\mathfrak{g}_{1}, \mathfrak{g}_{1}\right)$ and $l_{X}: \mathfrak{g}_{0} \wedge \mathfrak{g}_{0} \rightarrow \mathfrak{g}_{1}$ is a linear map, such that for any $x, y, z \in \mathfrak{g}_{0}, a \in \mathfrak{g}_{1}$,

$$
\left\{\begin{aligned}
d \circ X_{1} & =X_{0} \circ d \\
d l_{X}(x, y) & =X[x, y]-[X x, y]-[x, X y] \\
l_{X}(x, d a) & =X[x, a]-[X x, a]-[x, X a] \\
X l_{3}(x, y, z) & =l_{X}(x,[y, z])+\left[x, l_{X}(y, z)\right]+l_{3}(X x, y, z)+c . p \cdot(x, y, z) .
\end{aligned}\right.
$$

Denote by $\operatorname{Der}_{0}(\mathfrak{g})$ the set of derivations of degree 0 of $\mathfrak{g}$. Then one can define a 2 -vector space as

$$
\operatorname{Der}(\mathfrak{g}): \operatorname{Der}_{1}(\mathfrak{g}) \triangleq \operatorname{Hom}\left(\mathfrak{g}_{0}, \mathfrak{g}_{1}\right) \stackrel{\bar{d}}{\longrightarrow} \operatorname{Der}_{0}(\mathfrak{g})
$$


where $\bar{d}$ is given by $\bar{d}(\Theta)=\delta(\Theta)+l_{\delta(\Theta)}$, in which $\delta(\Theta)=d \circ \Theta+\Theta \circ d$ and

$$
l_{\delta(\Theta)}(x, y)=\Theta[x, y]-[x, \Theta y]-[\Theta x, y] .
$$

In addition, define $\left\{X+l_{X}, \Theta\right\}=[X, \Theta]$ and

$$
\left\{X+l_{X}, Y+l_{Y}\right\}=[X, Y]+X \triangleright l_{Y}-Y \triangleright l_{X},
$$

where $[\cdot, \cdot]$ is the commutator bracket and

$$
X \triangleright l_{Y}(x, y)=X l_{Y}(x, y)-l_{Y}(X x, y)-l_{Y}(x, X y) .
$$

Theorem 2.6. [5] With notations above, $(\operatorname{Der}(\mathfrak{g}),\{\cdot, \cdot\})$ is a strict Lie 2-algebra.

Remark 2.7. From the homological viewpoint, we discover an alternative description of $\operatorname{Der}_{0}(\mathfrak{g})$ and $\bar{d}$. Comparing Equations (11) and $\bar{d}$ with (4)-(7) and (9) respectively, we note that $\operatorname{Der}_{0}(\mathfrak{g})$ is indeed the set of 1-cocycles of the Lie 2-algebra $\mathfrak{g}$ with respect to the adjoint action and $\bar{d}=-\mathrm{D}$, which $\mathrm{D}$ is the Lie 2-algebra coboundary operator.

The adjoint action $a d$ can be extended to a new Lie 2-algebra homomorphism $\overline{a d}: \mathfrak{g} \longrightarrow \operatorname{Der}(\mathfrak{g})$, where

$$
\overline{a d}_{0}(x)=-\mathrm{D}(x)=a d_{0}(x)+l_{3}(x, \cdot, \cdot), \quad \overline{a d}_{1}=a d_{1}, \quad \overline{a d}_{2}=a d_{2}, \quad \forall x \in \mathfrak{g}_{0} .
$$

We conclude this section by exploring the derivations of a skeleton Lie 2-algebra, which turns out to have an explicit homological description.

Example 2.8. Let $\mathfrak{g}_{0}$ be an Lie algebra and $V$ a $\mathfrak{g}_{0}$-module. Given an Lie algebra 3-cocycle $l_{3} \in C^{3}\left(\mathfrak{g}_{0}, V\right)$, we get a skeletal Lie 2-algebra $\mathfrak{g}=\left(V \stackrel{0}{\longrightarrow} \mathfrak{g}_{0}, l_{2}, l_{3}\right)$, where $l_{2}$ is defined by

$$
l_{2}^{0}(x, y)=[x, y]_{\mathfrak{g}_{0}}, \quad l_{2}^{1}(x, u)=x \triangleright u, \quad \forall x, y \in \mathfrak{g}_{0}, u \in V .
$$

For $X=\left(X_{0}, X_{1}\right) \in \operatorname{Hom}\left(\mathfrak{g}_{0}, \mathfrak{g}_{0}\right) \oplus \operatorname{Hom}(V, V)$ and $l_{X} \in C^{2}\left(\mathfrak{g}_{0}, V\right)$, it is easy to check that

$$
X+l_{X} \in \operatorname{Der}_{0}(\mathfrak{g}) \Longleftrightarrow\left\{\begin{aligned}
X & \in \operatorname{Der}\left(\mathfrak{g}_{0} \ltimes V\right), \\
\mathfrak{D} l_{X} & =\left[X, l_{3}\right],
\end{aligned}\right.
$$

where $\mathfrak{D}: C^{k}\left(\mathfrak{g}_{0}, V\right) \rightarrow C^{k+1}\left(\mathfrak{g}_{0}, V\right)$ is the Lie algebra coboundary operator, Der $\left(\mathfrak{g}_{0} \ltimes V\right)$ is the Lie algebra of derivations of the semi-product Lie algebra $\mathfrak{g}_{0} \ltimes V$ and the bracket $[\cdot, \cdot]$ is given by

$$
\left[X, l_{3}\right](x, y, z)=X_{1} l_{3}(x, y, z)-l_{3}\left(X_{0} x, y, z\right)-l_{3}\left(x, X_{0} y, z\right)-l_{3}\left(x, y, X_{0} z\right), \quad \forall x, y, z \in \mathfrak{g}_{0} .
$$

In fact, such a bracket was used to introduce the notion of pre-Lie algebras by Gerstenhaber in 10. In this case, we have $\operatorname{Der}_{1}(\mathfrak{g})=C^{1}\left(\mathfrak{g}_{0}, V\right)$ and the map $\bar{d}: \operatorname{Der}_{1}(\mathfrak{g}) \rightarrow \operatorname{Der}_{0}(\mathfrak{g})$ is given by $\bar{d}(\Theta)=0-\mathfrak{D}(\Theta)$.

\section{Crossed modules of Lie 2-algebras}

\subsection{Definition of crossed modules}

Let $\left(\mathfrak{m}: \mathfrak{m}_{1} \stackrel{\tilde{d}}{\longrightarrow} \mathfrak{m}_{0}, \tilde{l}_{2}, \tilde{l}_{3}\right)$ and $\left(\mathfrak{g}: \mathfrak{g}_{1} \stackrel{d}{\longrightarrow} \mathfrak{g}_{0}, l_{2}, l_{3}\right)$ be two Lie 2 -algebras. We call $\mathfrak{g}$ acts on $\mathfrak{m}$ by derivations if there exists a Lie 2-algebra homomorphism $\phi: \mathfrak{g} \longrightarrow$ End( $\mathfrak{m})$ and a linear map $l_{\phi_{0}(x)}: \wedge^{2} \mathfrak{m}_{0} \longrightarrow \mathfrak{m}_{1}$ such that $\phi_{0}(x)+l_{\phi_{0}(x)} \in \operatorname{Der}_{0}(\mathfrak{m})$ and the map

$$
\left(\phi_{0}+l_{\phi_{0}}, \phi_{1}, \phi_{2}\right): \mathfrak{g} \longrightarrow \operatorname{Der}(\mathfrak{m})
$$

is a Lie 2-algebra homomorphism. By abuse of notations, we denote by $\phi$ both the action and the action by derivations. Then we shall define a crossed product of $\mathfrak{g}$ and $\mathfrak{m}$ denoted by $\mathfrak{g} \triangleright_{\phi} \mathfrak{m}$, which is still a Lie 2-algebra depending on the following lemma. 
Lemma 3.1. Let $\phi$ be an action of $\mathfrak{g}$ on $\mathfrak{m}$ by derivations, then $\mathfrak{g} \triangleright_{\phi} \mathfrak{m} \triangleq\left(\mathfrak{g} \oplus \mathfrak{m}, L_{1},[\cdot, \cdot], L_{3}\right)$ is a Lie 2-algebra with $\mathfrak{g}$ as a lie 2-subalgebra and $\mathfrak{m}$ as an ideal, where $L_{1}=d+\tilde{d}$ and

$$
\left\{\begin{aligned}
{[x+\alpha, y+\beta]=} & l_{2}(x, y)+\tilde{l}_{2}(\alpha, \beta)+x \triangleright \beta-y \triangleright \alpha, \quad \forall x, y \in \mathfrak{g}, \forall \alpha, \beta \in \mathfrak{m}, \\
L_{3}(x+\alpha, y+\beta, z+\gamma)= & l_{3}(x, y, z)+\tilde{l}_{3}(\alpha, \beta, \gamma)-(x, y) \triangleright \gamma-(y, z) \triangleright \alpha \\
& -(z, x) \triangleright \beta+l_{\phi_{0}(x)}(\beta, \gamma)+l_{\phi_{0}(y)}(\gamma, \alpha)+l_{\phi_{0}(z)}(\alpha, \beta), \\
& \forall x, y, z \in \mathfrak{g}_{0}, \forall \alpha, \beta, \gamma \in \mathfrak{m}_{0} .
\end{aligned}\right.
$$

Conversely, let $\left(\theta, L_{1}, L_{2}, L_{3}\right)$ be a Lie 2-algebra which can be split into the direct sum of a Lie 2 -subalgebra $\mathfrak{g}$ and an ideal $\mathfrak{m}$, then there exists an action $\phi$ of $\mathfrak{g}$ on $\mathfrak{m}$ by derivations such that $\theta=\mathfrak{g} \triangleright_{\phi} \mathfrak{m}$, where $\phi: \mathfrak{g} \longrightarrow \operatorname{Der}(\mathfrak{m})$ is defined by

$$
\left\{\begin{aligned}
\phi_{0}(x)+l_{\phi_{0}(x)} & =L_{2}(x, \cdot)+L_{3}(x, \cdot, \cdot), & & \forall x \in \mathfrak{g}_{0}, \\
\phi_{1}(a) & =L_{2}(a, \cdot), & & \forall a \in \mathfrak{g}_{1}, \\
\phi_{2}(x, y) & =-L_{3}(x, y, \cdot), & & \forall x, y \in \mathfrak{g}_{0} .
\end{aligned}\right.
$$

Proof. We merely provide the proof of the coherence law of $L_{2}, L_{3}$. Denote by $[\cdot, \cdot]$ both $l_{2}$ and $\tilde{l}_{2}$ if there is no risk of confusion. Firstly, for four elements in $\mathfrak{g}_{0}$ or four elements in $\mathfrak{m}_{0}$, it holds since $\mathfrak{g}$ and $\mathfrak{m}$ are Lie 2 -algebras and $L_{2}, L_{3}$ reserve their brackets. For any $x, y, z \in \mathfrak{g}_{0}, \alpha, \beta, \gamma \in \mathfrak{m}_{0}$, we have

$$
\begin{aligned}
& {\left[L_{3}(x, \alpha, \beta), \gamma\right]+L_{3}(x,[\alpha, \beta], \gamma)-L_{3}([x, \alpha], \beta, \gamma)+c . p \cdot(\alpha, \beta, \gamma)-\left[L_{3}(\alpha, \beta, \gamma), x\right] } \\
= & {\left[l_{\phi_{0}(x)}(\alpha, \beta), \gamma\right]+l_{\phi_{0}(x)}([\alpha, \beta], \gamma)-\tilde{l}_{3}(x \triangleright \alpha, \beta, \gamma)+c . p \cdot(\alpha, \beta, \gamma)+x \triangleright \tilde{l}_{3}(\alpha, \beta, \gamma), }
\end{aligned}
$$

which vanishes since $\phi_{0}(x)+l_{\phi_{0}(x)} \in \operatorname{Der}_{0}(\mathfrak{m})$. Next, making use of the fact $\phi$ is an action by derivations coupled with (12), we have

$$
l_{\phi_{0}[x, y]}-\left(\phi_{0}(x) \triangleright l_{\phi_{0}(y)}-\phi_{0}(y) \triangleright l_{\phi_{0}(x)}\right)=l_{\delta \phi_{2}(x, y)} .
$$

Hence,

$$
\begin{aligned}
& -\left[L_{3}(y, \alpha, \beta), x\right]+L_{3}([x, \alpha], y, \beta)-L_{3}([x, \beta], y, \alpha)-c . p .(x, y) \\
& +\left[L_{3}(x, y, \alpha), \beta\right]-\left[L_{3}(\beta, x, y), \alpha\right]-L_{3}([\alpha, \beta], x, y)-L_{3}([x, y], \alpha, \beta) \\
= & x \triangleright l_{\phi_{0}(y)}(\alpha, \beta)-l_{\phi_{0}(y)}(x \triangleright \alpha, \beta)-l_{\phi_{0}(y)}(\alpha, x \triangleright \beta)-c . p .(x, y) \\
& -[(x, y) \triangleright \alpha, \beta]+[(x, y) \triangleright \beta, \alpha]+(x, y) \triangleright[\alpha, \beta]-l_{\phi_{0}[x, y]}(\alpha, \beta) \\
= & \left(\phi_{0}(x) \triangleright l_{\phi_{0}(y)}-\phi_{0}(y) \triangleright l_{\phi_{0}(x)}\right)(\alpha, \beta)+l_{\delta \phi_{2}(x, y)}(\alpha, \beta)-l_{\phi_{0}[x, y]}(\alpha, \beta) \\
= & 0 .
\end{aligned}
$$

Now, it remains to show

$$
\begin{aligned}
& -\left[L_{3}(y, z, \alpha), x\right]-L_{3}([x, \alpha], y, z)-L_{3}([x, y], z, \alpha)+\text { c.p. }(x, y, z)+\left[L_{3}(x, y, z), \alpha\right] \\
= & -x \triangleright((y, z) \triangleright \alpha)+(y, z) \triangleright(x \triangleright \alpha)+([x, y], z) \triangleright \alpha+c \cdot p \cdot(x, y, z)+\left[L_{3}(x, y, z), \alpha\right] \\
= & \left(\left[\phi_{2}(y, z), \phi_{0}(x)\right]+\phi_{2}([x, y], z)+c \cdot p \cdot(x, y, z)+\phi_{1}\left(l_{3}(x, y, z)\right)\right) \alpha \\
= & 0,
\end{aligned}
$$

where the last equality follows from that $\phi$ is a Lie 2-algebra homomorphism. This finishes the proof of $\mathfrak{g} \triangleright_{\phi} \mathfrak{m}$ is Lie 2-algebra. The remaining results are easy to get.

Definition 3.2. A crossed module of Lie 2-algebras is a quadruple $(\mathfrak{m}, \mathfrak{g}, \phi, \Pi)$, where $\mathfrak{m}, \mathfrak{g}$ are two Lie 2-algebras, $\phi$ is an action of $\mathfrak{g}$ on $\mathfrak{m}$ by derivations, and $\Pi: \mathfrak{g} \triangleright_{\phi} \mathfrak{m} \rightarrow \mathfrak{g}$ is a Lie 2-algebra homomorphism, such that $\left.\Pi\right|_{\mathfrak{g}}=I d=(i d, i d, 0)$ and 
(i) $\tilde{l}_{2}(\alpha, \beta)=\Pi(\alpha) \triangleright \beta, \quad \forall \alpha, \beta \in \mathfrak{m}$,

(ii) $\tilde{l}_{3}(\alpha, \beta, \gamma)=-\left(\Pi_{0} \alpha, \Pi_{0} \beta\right) \triangleright \gamma-\Pi_{2}\left(\Pi_{0} \alpha, \beta\right) \triangleright \gamma, \quad \forall \alpha, \beta, \gamma \in \mathfrak{m}_{0}$,

(iii) $l_{\phi_{0}(x)}(\beta, \gamma)=-\left(x, \Pi_{0} \beta\right) \triangleright \gamma-\Pi_{2}(x, \beta) \triangleright \gamma, \quad \forall \beta, \gamma \in \mathfrak{m}_{0}, x \in \mathfrak{g}_{0}$,

(iv) $\Pi_{2}(\alpha, \beta)=\Pi_{2}\left(\Pi_{0} \alpha, \beta\right)=\Pi_{2}\left(\alpha, \Pi_{0} \beta\right), \quad \forall \alpha, \beta \in \mathfrak{m}_{0}$.

In particular, it is called a strong crossed module of Lie 2-algebras if $\Pi_{2}=0$.

We drop the words "Lie 2-algebras" except when emphasis is needed. For a crossed module $(\mathfrak{m}, \mathfrak{g}, \phi, \Pi)$, decompose $\Pi$ into

$$
\Pi=\left(\Pi_{0}, \Pi_{1}, \Pi_{2}\right)=I d+\sigma+\varphi=\left(\left(i d, \varphi_{0}\right),\left(i d, \varphi_{1}\right),\left(0, \sigma, \varphi_{2}\right)\right)
$$

where $\varphi=\left.\Pi\right|_{\mathfrak{m}}$ and $\sigma=\left.\Pi_{2}\right|_{\mathfrak{g}_{0} \wedge \mathfrak{m}_{0}}$. It is evident that $\varphi: \mathfrak{m} \longrightarrow \mathfrak{g}$ is a Lie 2-algebra homomorphism. In the following, we always describe a crossed module as $(\mathfrak{m}, \mathfrak{g}, \phi, \varphi, \sigma)$. Then a strong crossed module means $\sigma=0$ and is denoted by $(\mathfrak{m}, \mathfrak{g}, \phi, \varphi)$.

Definition 3.3. Let $(\mathfrak{m}, \mathfrak{g}, \phi, \varphi, \sigma)$ and $\left(\mathfrak{m}^{\prime}, \mathfrak{g}^{\prime}, \phi^{\prime}, \varphi^{\prime}, \sigma^{\prime}\right)$ be crossed modules, a morphism of crossed modules consists of two Lie 2-algebra homomorphisms $F: \mathfrak{m} \longrightarrow \mathfrak{m}^{\prime}, G: \mathfrak{g} \longrightarrow \mathfrak{g}^{\prime}$ and a linear map $\tau: \mathfrak{g}_{0} \wedge \mathfrak{m}_{0} \longrightarrow \mathfrak{m}_{1}^{\prime}$ such that $\varphi^{\prime} \circ F=G \circ \varphi$ and

$$
\left(\left(G_{0}, F_{0}\right),\left(G_{1}, F_{1}\right),\left(G_{2}, \tau, F_{2}\right)\right): \mathfrak{g} \triangleright_{\phi} \mathfrak{m} \longrightarrow \mathfrak{g}^{\prime} \triangleright_{\phi^{\prime}} \mathfrak{m}^{\prime}
$$

is a homomorphism of Lie 2-algebras. If $G_{2}, F_{2}, \tau$ vanish, we call it a strong morphism.

Similar to the Lie algebra case, we have the following proposition.

Proposition 3.4. Let $\mathfrak{g}$ be a Lie 2-algebra and $\mathfrak{m}$ a $\mathfrak{g}$-module. Given a chain map $\varphi: \mathfrak{m} \rightarrow \mathfrak{g}$ and a map $\sigma: \mathfrak{g}_{0} \wedge \mathfrak{m}_{0} \longrightarrow \mathfrak{g}_{1}$, satisfying that

$$
\Pi \triangleq\left(\left(i d, \varphi_{0}\right),\left(i d, \varphi_{1}\right),(0, \sigma, 0)\right): \mathfrak{g} \ltimes_{\phi} \mathfrak{m} \longrightarrow \mathfrak{g}
$$

is a Lie 2-algebra homomorphism and

(1) $\varphi(\alpha) \triangleright \beta=-\varphi(\beta) \triangleright \alpha, \quad \forall \alpha, \beta \in \mathfrak{m}$,

(2) $\left(\varphi_{0} \alpha, \varphi_{0} \beta\right) \triangleright \gamma+\sigma\left(\varphi_{0} \alpha, \beta\right) \triangleright \gamma=-\left(\varphi_{0} \alpha, \varphi_{0} \gamma\right) \triangleright \beta-\sigma\left(\varphi_{0} \alpha, \gamma\right) \triangleright \beta, \quad \forall \alpha, \beta, \gamma \in \mathfrak{m}_{0}$,

(3) $\left(x, \varphi_{0} \beta\right) \triangleright \gamma+\sigma(x, \beta) \triangleright \gamma=-\left(x, \varphi_{0} \gamma\right) \triangleright \beta-\sigma(x, \gamma) \triangleright \beta, \quad \forall \beta, \gamma \in \mathfrak{m}_{0}, x \in \mathfrak{g}_{0}$,

(4) $\sigma\left(\varphi_{0} \alpha, \beta\right)=\sigma\left(\alpha, \varphi_{0} \beta\right), \quad \forall \alpha, \beta \in \mathfrak{m}_{0}$,

then, there exists a unique Lie 2-algebra structure on $\mathfrak{m}$, linear maps $l_{\phi_{0}(x)}: \wedge^{2} \mathfrak{m}_{0} \longrightarrow \mathfrak{m}_{1}$ and $\varphi_{2}: \wedge^{2} \mathfrak{m}_{0} \longrightarrow \mathfrak{g}_{1}$ such that $(\mathfrak{m}, \mathfrak{g}, \hat{\phi}, \hat{\varphi}, \sigma)$ is a crossed module, where $\hat{\phi}=\left(\phi_{0}+l_{\phi_{0}}, \phi_{1}, \phi_{2}\right)$ and $\hat{\varphi}=\left(\varphi_{0}, \varphi_{1}, \varphi_{2}\right)$.

Proof. Define $\tilde{l}_{2}, \tilde{l}_{3}, l_{\phi_{0}(x)}, \varphi_{2}$ on $\mathfrak{m}$ by the right hand sides of equalities $(i)$-(iv) of Definition 3.2 Then, by direct verification, we obtain that $\mathfrak{m}$ with $\tilde{l}_{2}, \tilde{l}_{3}$ is a Lie 2 -algebra and $\hat{\phi}: \mathfrak{g} \longrightarrow \operatorname{Der}(\mathfrak{m})$ is a Lie 2-algebra homomorphism. Moreover, $\hat{\Pi}=\left(\left(i d, \varphi_{0}\right),\left(i d, \varphi_{1}\right),\left(0, \sigma, \varphi_{2}\right)\right): \mathfrak{g} \triangleright_{\hat{\phi}} \mathfrak{m} \longrightarrow \mathfrak{g}$ is a Lie 2 -algebra homomorphism. Thus, we get a crossed module of Lie 2 -algebras. 


\subsection{Lie 3-algebras associated to crossed modules}

A crossed module $(\mathfrak{m}, \mathfrak{g}, \phi, \varphi, \sigma)$ corresponds to a square

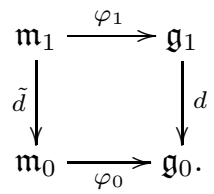

Consider its mapping cone complex ([13])

$$
\mathfrak{V}: \mathfrak{m}_{1} \stackrel{d_{D}}{\longrightarrow} \mathfrak{g}_{1} \oplus \mathfrak{m}_{0} \stackrel{d_{D}}{\longrightarrow} \mathfrak{g}_{0},
$$

where

$$
d_{D}(\xi)=-\varphi_{1} \xi+\tilde{d} \xi, \quad d_{D}(a+\alpha)=d a+\varphi_{0} \alpha, \quad \forall \xi \in \mathfrak{m}_{1}, a+\alpha \in \mathfrak{g}_{1} \oplus \mathfrak{m}_{0} .
$$

Then $d_{D}^{2}=0$ follows from the fact that $d \circ \varphi_{1}=\varphi_{0} \circ \tilde{d}$.

Define $\llbracket \cdot, \cdot \rrbracket$ and $l^{3}$ on it by: for any $x, y, z \in \mathfrak{g}_{0}, a, b \in \mathfrak{g}_{1}, \alpha, \beta \in \mathfrak{m}_{0}$ and $\xi \in \mathfrak{m}_{1}$,

$$
\left\{\begin{aligned}
\llbracket x, y \rrbracket & =l_{2}(x, y), \\
\llbracket x, \xi \rrbracket & =-\llbracket \xi, x \rrbracket=x \triangleright \xi, \\
\llbracket a+\alpha, b+\beta \rrbracket & =a \triangleright \beta+b \triangleright \alpha, \\
\llbracket x, a+\alpha \rrbracket & =-\llbracket a+\alpha, x \rrbracket=l_{2}(x, a)-\sigma(x, \alpha)+x \triangleright \alpha, \\
l^{3}(x, y, z) & =l_{3}(x, y, z), \\
l^{3}(x, y, a+\alpha) & =-l^{3}(x, a+\alpha, y)=l^{3}(a+\alpha, x, y)=-(x, y) \triangleright \alpha .
\end{aligned}\right.
$$

Theorem 3.5. With the above notations, $\left(\mathfrak{V}, d_{D}, \llbracket \cdot, \cdot \rrbracket, l^{3}\right)$ is a strict Lie 3 -algebra.

Proof. We need to verify all the conditions of Lie 3 -algebras. Firstly, it is obvious that $\llbracket \cdot, \cdot \rrbracket$ and $l^{3}$ are antisymmetric in the graded sense. Then, it suffices to prove that equality (1) of Definition 2.1 holds for $1 \leq n \leq 5$.

- $n=1$ : (1) reduces to $d_{D}^{2}=0$, which has already been checked.

- $n=2$ : Condition (1) gives

$$
d_{D}[x, y]=\llbracket d_{D} x, y \rrbracket+(-1)^{|x|} \llbracket x, d_{D} y \rrbracket, \quad \forall x, y \in \mathfrak{V},
$$

which is equivalent to

$$
\left\{\begin{aligned}
d_{D} \llbracket x, \xi \rrbracket & =\llbracket x, d_{D} \xi \rrbracket \\
d_{D} \llbracket x, a+\alpha \rrbracket & =\llbracket x, d_{D}(a+\alpha) \rrbracket, \\
\llbracket d_{D}(a+\alpha), \xi \rrbracket & =\llbracket a+\alpha, d_{D} \xi \rrbracket, \\
d_{D} \llbracket a+\alpha, b+\beta \rrbracket & =\llbracket d_{D}(a+\alpha), b+\beta \rrbracket-\llbracket a+\alpha, d_{D}(b+\beta) \rrbracket .
\end{aligned}\right.
$$

The first three equations are easy to verify. As for the last one, by direct computations, we have

$$
\begin{aligned}
d_{D} \llbracket a+\alpha, b+\beta \rrbracket & =d_{D}(a \triangleright \beta+b \triangleright \alpha) \\
& =-\varphi_{1}(a \triangleright \beta)-\varphi_{1}(b \triangleright \alpha)+\tilde{d}(a \triangleright \beta)+\tilde{d}(b \triangleright \alpha),
\end{aligned}
$$

and

$$
\begin{aligned}
& \llbracket d_{D}(a+\alpha), b+\beta \rrbracket-\llbracket a+\alpha, d_{D}(b+\beta) \rrbracket \\
= & {\left[d a+\varphi_{0} \alpha, b\right]-\sigma\left(d a+\varphi_{0} \alpha, \beta\right)+\left(d a+\varphi_{0} \alpha\right) \triangleright \beta } \\
& +\left[d b+\varphi_{0} \beta, a\right]-\sigma\left(d b+\varphi_{0} \beta, \alpha\right)+\left(d b+\varphi_{0} \beta\right) \triangleright \alpha \\
= & {\left[\varphi_{0} \alpha, b\right]+\sigma(\alpha, d b)-\left[a, \varphi_{0} \beta\right]-\sigma(d a, \beta)+d a \triangleright \beta+d b \triangleright \alpha, }
\end{aligned}
$$


where we have used conditions $(i)$ and (iv) of Definition 3.2. Therefore, the equality $d_{D} \llbracket a+\alpha, b+\beta \rrbracket=$ $\llbracket d_{D}(a+\alpha), b+\beta \rrbracket-\llbracket a+\alpha, d_{D}(b+\beta) \rrbracket$ holds since $\Pi$ is a homomorphism.

- $n=3$ : We are supposed to check the graded Jacobi identity: for any $x, y, z \in \mathfrak{V}$,

$$
\begin{aligned}
& (-1)^{|x| \cdot|z|} \llbracket \llbracket x, y \rrbracket, z \rrbracket+c . p . \\
= & (-1)^{|x| \cdot|z|+1}\left\{d_{D} l^{3}(x, y, z)+l^{3}\left(d_{D} x, y, z\right)+(-1)^{|x|} l^{3}\left(x, d_{D} y, z\right)+(-1)^{|x|+|y|} l^{3}\left(x, y, d_{D} z\right)\right\} .
\end{aligned}
$$

Following from that $\triangleright$ is an action and $\Pi$ is a homomorphism, we have

$$
\begin{gathered}
\llbracket \llbracket x, y \rrbracket, z \rrbracket+c . p .=-d l_{3}(x, y, z)=-d_{D} l^{3}(x, y, z), \\
\llbracket \llbracket x, y \rrbracket, \xi \rrbracket+c . p .=(x, y) \triangleright \tilde{d} \xi=-l^{3}\left(x, y, d_{D} \xi\right),
\end{gathered}
$$

and

$$
\begin{aligned}
& \llbracket \llbracket x, y \rrbracket, a+\alpha \rrbracket+c . p . \\
= & {[[x, y], a]-\sigma([x, y], \alpha)+[x, y] \triangleright \alpha+[[y, a], x]-[\sigma(y, \alpha), x]-\sigma(y \triangleright \alpha, x)-x \triangleright(y \triangleright \alpha) } \\
& -[[x, a], y]+[\sigma(x, \alpha), y]+\sigma(x \triangleright \alpha, y)+y \triangleright(x \triangleright \alpha) \\
= & -l_{3}(x, y, d a)+\tilde{d}((x, y) \triangleright \alpha)-\varphi_{1}((x, y) \triangleright \alpha)-l_{3}\left(x, y, \varphi_{0} \alpha\right) \\
= & -l^{3}\left(x, y, d_{D}(a+\alpha)\right)-d_{D} l^{3}(x, y, a+\alpha) .
\end{aligned}
$$

The next case is,

$$
\begin{aligned}
& \llbracket \llbracket x, a+\alpha \rrbracket, b+\beta \rrbracket+\llbracket \llbracket a+\alpha, b+\beta \rrbracket, x \rrbracket-\llbracket \llbracket b+\beta, x \rrbracket, a+\alpha \rrbracket \\
= & {[x, a] \triangleright \beta-\sigma(x, \alpha) \triangleright \beta+b \triangleright(x \triangleright \alpha)-x \triangleright(a \triangleright \beta)-x \triangleright(b \triangleright \alpha) } \\
& +[x, b] \triangleright \alpha-\sigma(x, \beta) \triangleright \alpha+a \triangleright(x \triangleright \beta) \\
= & (x, d a) \triangleright \beta+(x, d b) \triangleright \alpha+\left(x, \varphi_{0} \alpha\right) \triangleright \beta+\left(x, \varphi_{0} \beta\right) \triangleright \alpha \\
= & -l^{3}\left(x, d_{D}(a+\alpha), b+\beta\right)+l^{3}\left(x, a+\alpha, d_{D}(b+\beta)\right),
\end{aligned}
$$

where we have used the equation $\sigma(x, \alpha) \triangleright \beta+\sigma(x, \beta) \triangleright \alpha=-\left(x, \varphi_{0} \alpha\right) \triangleright \beta-\left(x, \varphi_{0} \beta\right) \triangleright \alpha$ followed from condition (iii) of Definition 3.2, This finishes the proof of the graded Jacobi identity.

- $n=4$ : Specifically, for four elements in $\mathfrak{V}_{0},(1)$ holds since $\mathfrak{g}$ is a Lie 2-algebra and the definition of $l^{3}$. While for three elements in $\mathfrak{V}_{0}$ and one element in $\mathfrak{V}_{1}$, by straightforward deduce, condition (1) is equivalent to the coherence law of $L_{2}$ and $L_{3}$ on three elements in $\mathfrak{g}_{0}$ and one element in $\mathfrak{m}_{0}$ in Lemma 3.1. By careful analysis, all the other cases are trivial.

- $n=5$ : We shall prove

$$
\Sigma_{\sigma}(-1)^{\sigma} K \operatorname{sgn}(\sigma) l^{3}\left(l^{3}\left(x_{\sigma_{1}}, x_{\sigma_{2}}, x_{\sigma_{3}}\right), x_{\sigma_{4}}, x_{\sigma_{5}}\right)=0 .
$$

Actually, every term in the summation vanishes by the definition of $l^{3}$. This completes the proof

\subsection{Examples}

Example 3.6. Let $(\mathfrak{m}, \mathfrak{g}, \phi, \varphi)$ be a strong crossed module with strict Lie 2-algebras $\mathfrak{m}, \mathfrak{g}$ and strong homomorphism $\phi$. Treating $\mathfrak{g}$ as a crossed module of Lie algebras with $[a, b]=[d a, b]$ on $\mathfrak{g}_{1}$ and $\mathfrak{m}$ likewise, we get a commutative diagram

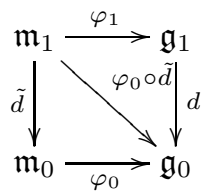


such that all the maps are crossed modules of Lie algebras. Moreover, defining $[a, \xi]=d a \triangleright \xi, \forall a \in$ $\mathfrak{g}_{1}, \xi \in \mathfrak{m}_{1}$, the crossed product $\mathfrak{g} \triangleright_{\phi} \mathfrak{m}$ is also a crossed module of Lie algebras.

Remark 3.7. This example remains us of the notion of crossed squares and 2-crossed modules of Lie algebras introduced by Ellis in [8]. The group-theoretic setting is due to Conduché ([77]). See also [20, 9] for more details. The relation between the strong crossed modules in the above example and crossed squares of Lie algebras is still a mystery to us, which deserves to be further studied.

Example 3.8. For a Lie 2-algebra $\left(\mathfrak{g}, d, l_{2}, l_{3}\right)$, it is obvious that $\operatorname{Der}(\mathfrak{g})$ acts on $\mathfrak{g}$ by derivations with $I d: \operatorname{Der}(\mathfrak{g}) \longrightarrow \operatorname{Der}(\mathfrak{g})$. Consider the adjoint homomorphism $\overline{a d}: \mathfrak{g} \longrightarrow \operatorname{Der}(\mathfrak{g})$ given by (13) and a linear map $\sigma: \operatorname{Der}_{0}(\mathfrak{g}) \wedge \mathfrak{g}_{0} \longrightarrow \operatorname{Der}_{1}(\mathfrak{g})$ defined by

$$
\sigma\left(X+l_{X}, x\right)=-l_{X}(x, \cdot), \quad \forall X+l_{X} \in \operatorname{Der}_{0}(\mathfrak{g}), x \in \mathfrak{g}_{0} .
$$

By straightforward verification, we get $(\mathfrak{g}, \operatorname{Der}(\mathfrak{g}), I d, \overline{a d}, \sigma)$ is a crossed module. Note that this crossed module is not strong even if $\mathfrak{g}$ is a strict Lie 2-algebra. From Theorem 3.5 it follows that the 3 -term complex of vector spaces

$$
\operatorname{DER}(\mathfrak{g}): \mathfrak{g}_{1} \stackrel{d_{D}}{\longrightarrow} \operatorname{Der}_{1}(\mathfrak{g}) \oplus \mathfrak{g}_{0} \stackrel{d_{D}}{\longrightarrow} \operatorname{Der}_{0}(\mathfrak{g})
$$

is a strict Lie 3-algebra. Moreover, $l^{3}$ vanishes by definition. This recovers [5, Theorem 3.8].

Example 3.9. Let $\left(\mathfrak{g}, d, l_{2}, l_{3}\right)$ be a Lie 2-algebra and $\mathfrak{m}$ an ideal of $\mathfrak{g}$, then $(\mathfrak{m}, \mathfrak{g}, \overline{a d}, i)$ is a strong crossed module, in which $\overline{a d}: \mathfrak{g} \longrightarrow \operatorname{Der}(\mathfrak{m})$ given by (13) is an action of $\mathfrak{g}$ on $\mathfrak{m}$ by derivations and the inclusion map $i: \mathfrak{m} \longrightarrow \mathfrak{g}$ is a strong homomorphism.

According to Example 3.8 and Example 3.9 with $\mathfrak{m}=\mathfrak{g}$, there are two natural crossed modules for a Lie 2-algebra $\mathfrak{g}$. Moreover, we get a commutative diagram

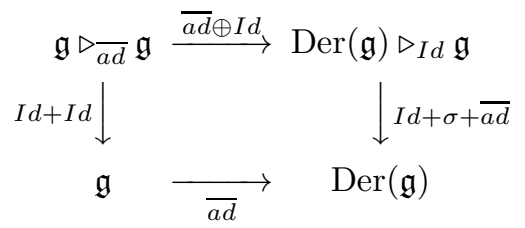

such that all the maps are Lie 2-algebra homomorphisms. Namely, $(I d, \overline{a d}, 0)$ is a homomorphism between the two crossed modules.

Example 3.10. The following example is inspired by [24, Example 3]. Given a Lie 2-algebra $\mathfrak{h}$, a short exact sequence of $\mathfrak{h}$-modules:

$$
0 \longrightarrow \mathbb{V} \stackrel{p}{\longrightarrow} \mathbb{I} \stackrel{q}{\longrightarrow} \mathbb{Q} \longrightarrow 0,
$$

(regarded as a short exact sequence of trivial Lie 2-algebras) and a 2-cocycle $\lambda \in C^{2}(\mathfrak{h}, \mathbb{Q})$, by $[15$, Theorem 4.5], we get an abelian extension of $\mathfrak{h}$ by $\mathbb{Q}$ :

$$
0 \rightarrow \mathbb{Q} \stackrel{i}{\longrightarrow} \mathfrak{h} \oplus_{\lambda} \mathbb{Q} \stackrel{\pi}{\longrightarrow} \mathfrak{h} \rightarrow 0 .
$$

Splicing (15) and (16) together, we get a strong crossed module $\varepsilon=\left(\mathbb{I}, \mathfrak{h} \oplus_{\lambda} \mathbb{Q}, \phi, \varphi\right)$, where $\mathbb{I} \stackrel{\varphi}{\longrightarrow} \mathfrak{h} \oplus_{\lambda} \mathbb{Q}$ is defined by $\varphi(v)=(0, q(v))$, and $\phi$ is an action of $\mathfrak{h} \oplus_{\lambda} \mathbb{Q}$ on $\mathbb{I}$ given by the action of $\mathfrak{h}$ on $\mathbb{I}$. Since $\mathbb{I}$ is a trivial Lie 2 -algebra, by defining $l_{\phi_{0}}=0$, it is clear that $\phi$ is an action by derivations. 


\section{The first cohomology and derivations of crossed modules}

\subsection{Lie algebra structures on $C^{1}(\mathfrak{g}, \mathbb{V})$}

Let $\mathbb{V}: V_{1} \stackrel{\partial}{\longrightarrow} V_{0}$ and $\mathbb{W}: W_{1} \stackrel{d}{\longrightarrow} W_{0}$ be two 2 -vector spaces. Then we can construct a new 2 -vector space

$$
\operatorname{Hom}(\mathbb{V}, \mathbb{W}): \operatorname{Hom}_{1}(\mathbb{V}, \mathbb{W}) \stackrel{\delta}{\longrightarrow} \operatorname{Hom}_{0}(\mathbb{V}, \mathbb{W})
$$

where $\operatorname{Hom}_{1}(\mathbb{V}, \mathbb{W})=\operatorname{Hom}\left(V_{0}, W_{1}\right)$,

$$
\operatorname{Hom}_{0}(\mathbb{V}, \mathbb{W})=\left\{X_{0}+X_{1} \in \operatorname{Hom}\left(V_{0}, W_{0}\right) \oplus \operatorname{Hom}\left(V_{1}, W_{1}\right) \mid X_{0} \circ \partial=d \circ X_{1}\right\},
$$

and $\delta(\Theta)=d \circ \Theta+\Theta \circ \partial$.

Lemma 4.1. Given $\varphi \in \operatorname{Hom}_{0}(\mathbb{W}, \mathbb{V})$, there exists a strict Lie 2-algebra structure on $\operatorname{Hom}(\mathbb{V}, \mathbb{W})$, where the bracket $[\cdot, \cdot]_{\varphi}$ is given by:

$$
[X, Y]_{\varphi}=X \circ \varphi \circ Y-Y \circ \varphi \circ X, \quad[X, \Theta]_{\varphi}=X \circ \varphi \circ \Theta-\Theta \circ \varphi \circ X, \quad\left[\Theta, \Theta^{\prime}\right]_{\varphi}=0 .
$$

In particular, if $\mathbb{W}=\mathbb{V}, \varphi=I d$, this recovers the strict Lie 2 -algebra $\operatorname{End}(\mathbb{V})$, which plays the same role as $\operatorname{gl}(V)$ for a vector space $V$.

Set $\mathrm{L}(\mathfrak{g}, \mathbb{V}) \triangleq \operatorname{Hom}\left(\mathfrak{g}_{0}, V_{0}\right) \oplus \operatorname{Hom}\left(\mathfrak{g}_{1}, V_{1}\right)$, then $C^{1}(\mathfrak{g}, \mathbb{V})=\mathrm{L}(\mathfrak{g}, \mathbb{V}) \oplus \operatorname{Hom}\left(\wedge^{2} \mathfrak{g}_{0}, V_{1}\right)$. Given an element $\varphi=\left(\varphi_{0}, \varphi_{1}\right) \in \operatorname{Hom}\left(V_{0}, \mathfrak{g}_{0}\right) \oplus \operatorname{Hom}\left(V_{1}, \mathfrak{g}_{1}\right)$, we have $\mathrm{L}(\mathfrak{g}, \mathbb{V})$ is a Lie algebra with the bracket $[\cdot, \cdot]_{\varphi}$ given by the first formula of (17). Furthermore, we have:

Lemma 4.2. (1) $\operatorname{Hom}\left(\wedge^{2} \mathfrak{g}_{0}, V_{1}\right)$ is an $\mathrm{L}(\mathfrak{g}, \mathbb{V})$-module, where the action is given by

$$
(X \triangleright \xi)(x, y)=X_{1} \varphi_{1} \xi(x, y)-\xi\left(\varphi_{0} X_{0} x, y\right)-\xi\left(x, \varphi_{0} X_{0} y\right), \quad \forall x, y \in \mathfrak{g}_{0},
$$

for any $X=\left(X_{0}, X_{1}\right) \in \mathrm{L}(\mathfrak{g}, \mathbb{V})$ and $\xi \in \operatorname{Hom}\left(\wedge^{2} \mathfrak{g}_{0}, V_{1}\right)$.

(2) let $\sigma: \mathfrak{g}_{0} \wedge V_{0} \longrightarrow \mathfrak{g}_{1}$ be a linear map satisfying $\sigma\left(\varphi_{0} u, v\right)=\sigma\left(u, \varphi_{0} v\right), \forall u, v \in V_{0}$. Then $\omega^{\sigma}$ defined by

$$
\omega^{\sigma}(X, Y)(x, y) \triangleq X \sigma(Y x, y)+X \sigma(x, Y y)-Y \sigma(X x, y)-Y \sigma(x, X y)
$$

is a 2-cocycle of $\mathrm{L}(\mathfrak{g}, \mathbb{V})$ with values in $\operatorname{Hom}\left(\wedge^{2} \mathfrak{g}_{0}, V_{1}\right)$.

(3) $C^{1}(\mathfrak{g}, \mathbb{V})$ is a Lie algebra with the bracket $\{\cdot, \cdot\}$ given by

$$
\{X+\xi, Y+\eta\}=[X, Y]_{\varphi}+X \triangleright \eta-Y \triangleright \xi+\omega^{\sigma}(X, Y) .
$$

Proof. To prove that $\triangleright$ is an action, we need to check

$$
[Y, X]_{\varphi} \triangleright \xi=Y \triangleright(X \triangleright \xi)-X \triangleright(Y \triangleright \xi) .
$$

It follows from

$$
\begin{aligned}
& (Y \triangleright(X \triangleright \xi)-X \triangleright(Y \triangleright \xi))(x, y) \\
= & Y \varphi(X \triangleright \xi)(x, y)-X \triangleright \xi(\varphi Y x, y)-X \triangleright \xi(x, \varphi Y y)-c . p \cdot(X, Y) \\
= & Y \varphi(X \varphi \xi(x, y)-\xi(\varphi X x, y)-\xi(x, \varphi X y))-X \varphi \xi(\varphi Y x, y)+\xi(\varphi X \varphi Y x, y)+\xi(\varphi Y x, \varphi X y) \\
& -X \varphi \xi(x, \varphi Y y)+\xi(\varphi X x, \varphi Y y)+\xi(x, \varphi X \varphi Y y)-c . p .(X, Y) \\
= & {[Y, X]_{\varphi} \varphi \xi(x, y)-\xi\left(\varphi[Y, X]_{\varphi} x, y\right)-\xi\left(x, \varphi[Y, X]_{\varphi} y\right) } \\
= & \left([Y, X]_{\varphi} \triangleright \xi\right)(x, y) .
\end{aligned}
$$

It is direct to verify that $\omega^{\sigma}$ is a 2 -cocycle. We omit the details. 


\subsection{Derivations of crossed modules}

The main goal of this section is to propose a notion of derivations of a crossed module of Lie 2-algebras and prove that it is a strict Lie 2-algebra, which generalises the derivations of a Lie 2-algebra $\operatorname{Der}(\mathfrak{g})$.

Given a crossed module $(\mathfrak{m}, \mathfrak{g}, \phi, \varphi, \sigma)$, since $\mathfrak{m}$ is a $\mathfrak{g}$-module, we have a natural complex

$$
\operatorname{Der}(\mathfrak{g}, \mathfrak{m}): \operatorname{Der}_{1}(\mathfrak{g}, \mathfrak{m}) \triangleq \operatorname{Hom}\left(\mathfrak{g}_{0}, \mathfrak{m}_{1}\right) \stackrel{-\mathrm{D}}{\longrightarrow} \operatorname{Der}_{0}(\mathfrak{g}, \mathfrak{m}),
$$

where $\operatorname{Der}_{0}(\mathfrak{g}, \mathfrak{m})$ is the set of 1-cocycles and D is the Lie 2-algebra coboundary operator. Denote by $\operatorname{Inn}_{0}(\mathfrak{g}, \mathfrak{m})$ the set of 1 -coboundaries. Explicitly, by (8) and (9), we have $-\mathrm{D}(\Theta)=\delta(\Theta)+l_{\delta(\Theta)}$ and

$$
l_{\delta(\Theta)}(x, y)=\Theta[x, y]-x \triangleright \Theta y+y \triangleright \Theta x, \quad \forall x, y \in \mathfrak{g}_{0}, \Theta \in \operatorname{Der}_{1}(\mathfrak{g}, \mathfrak{m}) .
$$

We call Der $(\mathfrak{g}, \mathfrak{m})$ the derivations of the crossed module.

By condition $(i v)$ of Definition 3.2 and Lemma 4.2, $\left(C^{1}(\mathfrak{g}, \mathfrak{m}),\{\cdot, \cdot\}\right)$ is a Lie algebra. As its subspace, $\operatorname{Der}_{0}(\mathfrak{g}, \mathfrak{m})$ inherits a bracket operation, that is, for any $X+l_{X}, Y+l_{Y} \in \operatorname{Der}_{0}(\mathfrak{g}, \mathfrak{m})$,

$$
\left\{X+l_{X}, Y+l_{Y}\right\}=[X, Y]_{\varphi}+X \triangleright l_{Y}-Y \triangleright l_{X}+\omega^{\sigma}(X, Y) .
$$

Set $l_{[X, Y]_{\varphi}}=X \triangleright l_{Y}-Y \triangleright l_{X}+\omega^{\sigma}(X, Y)$. To be more precise,

$$
l_{[X, Y]_{\varphi}}(x, y)=X \varphi l_{Y}(x, y)-l_{Y}(\varphi X x, y)-l_{Y}(x, \varphi X y)+X \sigma(Y x, y)+X \sigma(x, Y y)-\text { c.p. }(X, Y) .
$$

Define $\left\{X+l_{X}, \Theta\right\} \triangleq[X, \Theta]_{\varphi}$.

Lemma 4.3. With the above notations, $\operatorname{Der}_{0}(\mathfrak{g}, \mathfrak{m})$ is a Lie subalgebra of $\left(C^{1}(\mathfrak{g}, \mathfrak{m}),\{\cdot, \cdot\}\right)$.

Proof. For any $X+l_{X}, Y+l_{Y} \in \operatorname{Der}_{0}(\mathfrak{g}, \mathfrak{m})$, we need to prove that $[X, Y]_{\varphi}+l_{[X, Y]_{\varphi}}$ is a 1 -cocycle. It is not hard to check conditions (44)-(6). We just verify condition (7), i.e., for any $x, y, z \in \mathfrak{g}_{0}$,

$$
l_{[X, Y]_{\varphi}}(x,[y, z])+x \triangleright l_{[X, Y]_{\varphi}}(y, z)-(y, z) \triangleright[X, Y]_{\varphi} x+c \cdot p \cdot(x, y, z)=[X, Y]_{\varphi} l_{3}(x, y, z) .
$$

Firstly, as $X+l_{X}, Y+l_{Y} \in \operatorname{Der}_{0}(\mathfrak{g}, \mathfrak{m})$ and $\Pi=I d+\sigma+\varphi$ is a homomorphism, we have

$$
\begin{aligned}
\varphi X[y, z]= & \varphi\left(y \triangleright X z-z \triangleright X y+\tilde{d} l_{X}(y, z)\right) \\
= & {[y, \varphi X z]+d \sigma(y, X z)-[z, \varphi X y]-d \sigma(z, X y)+\varphi \tilde{d} l_{X}(y, z) } \\
X \varphi\left(Y l_{3}(x, y, z)\right)= & X \varphi\left(l_{Y}(x,[y, z])+x \triangleright l_{Y}(y, z)-(y, z) \triangleright Y x+c \cdot p \cdot(x, y, z)\right) \\
= & X \varphi l_{Y}(x,[y, z])+x \triangleright X \varphi l_{Y}(y, z)-\varphi l_{Y}(y, z) \triangleright X x+l_{X}\left(x, d \varphi l_{Y}(y, z)\right) \\
& +X \sigma\left(x, \tilde{d} l_{Y}(y, z)\right)-X \varphi((y, z) \triangleright Y x)+c . p .(x, y, z),
\end{aligned}
$$

and

$$
\begin{aligned}
& -X\left(\varphi((y, z) \triangleright Y x)+l_{3}(\varphi Y x, y, z)\right) \\
= & X(\sigma(Y x,[y, z])+\sigma(y, z \triangleright Y x)-\sigma(z, y \triangleright Y x)+[y, \sigma(z, Y x)]+[z, \sigma(Y x, y)]) \\
= & X \sigma(Y x,[y, z])+X \sigma(y, z \triangleright Y x)-X \sigma(z, y \triangleright Y x)+y \triangleright X \sigma(z, Y x)-\sigma(z, Y x) \triangleright X y \\
& +l_{X}(y, d \sigma(z, Y x))-z \triangleright X \sigma(y, Y x)+\sigma(y, Y x) \triangleright X z-l_{X}(z, d \sigma(y, Y x)) .
\end{aligned}
$$


Thus, (18) follows from

$$
\begin{aligned}
& l_{[X, Y]_{\varphi}}(x,[y, z])+x \triangleright l_{[X, Y]_{\varphi}}(y, z)-(y, z) \triangleright[X, Y]_{\varphi} x+c \cdot p \cdot(x, y, z) \\
= & X \varphi l_{Y}(x,[y, z])-l_{Y}\left(x,[y, \varphi X z]+d \sigma(y, X z)-[z, \varphi X y]-d \sigma(z, X y)+\varphi \tilde{d} l_{X}(y, z)\right) \\
& -l_{Y}(\varphi X x,[y, z])+X \sigma(Y x,[y, z])+X \sigma\left(x, y \triangleright Y z-z \triangleright Y y+\tilde{d} l_{Y}(y, z)\right) \\
& +x \triangleright\left(X \varphi l_{Y}(y, z)-l_{Y}(\varphi X y, z)-l_{Y}(y, \varphi X z)+X \sigma(Y y, z)+X \sigma(y, Y z)\right) \\
& -(y, z) \triangleright X \varphi Y x-c \cdot p \cdot(X, Y)+c \cdot p \cdot(x, y, z) \quad b y(19) \\
= & \left(X \varphi l_{Y}(x,[y, z])+x \triangleright X \varphi l_{Y}(y, z)+l_{X}\left(x, d \varphi l_{Y}(y, z)\right)+X \sigma\left(x, \tilde{d} l_{Y}(y, z)\right)\right) \\
& -\left(l_{Y}(\varphi X x,[y, z])+c \cdot p \cdot(\varphi X x, y, z)+y \triangleright l_{Y}(z, \varphi X x)+z \triangleright l_{Y}(\varphi X x, y)-(y, z) \triangleright Y \varphi X x\right) \\
& +\left(X \sigma(Y x,[y, z])+c \cdot p \cdot(Y x, y, z)+y \triangleright X \sigma(z, Y x)+l_{X}(y, d \sigma(z, Y x))\right. \\
& -z \triangleright X \sigma(y, Y x)-l_{X}(z, d \sigma(y, Y x))-c \cdot p \cdot(X, Y)+c \cdot p \cdot(x, y, z) \\
= & X \varphi Y l_{3}(x, y, z)+\left(\varphi l_{Y}(y, z) \triangleright X x+X \varphi((y, z) \triangleright Y x)\right. \\
& -Y l_{3}(\varphi X x, y, z)+\varphi X x \triangleright l_{Y}(y, z)-(z, \varphi X x) \triangleright Y y-(\varphi X x, y) \triangleright Y z \\
& \left.-X \varphi((y, z) \triangleright Y x)-X l_{3}(\varphi Y x, y, z)+\sigma(z, Y x) \triangleright X y-\sigma(y, Y x) \triangleright X z+c \cdot p \cdot(x, y, z)\right) \\
& -c \cdot p \cdot(X, Y) \quad b y(20),(7) \text { and (21) } \\
= & l_{\phi_{0}(z)}(X x, Y y)-l_{\phi_{0}(y)}(X x, Y z)+c . p \cdot(x, y, z)-c . p \cdot(X, Y)+[X, Y]_{\varphi} l_{3}(x, y, z) \\
= & {[X, Y]_{\varphi} l_{3}(x, y, z), }
\end{aligned}
$$

where the penultimate equation holds since conditions (i) and (iii) of Definition 3.2,

Theorem 4.4. For a crossed module $(\mathfrak{m}, \mathfrak{g}, \phi, \varphi, \sigma)$, its derivations $(\operatorname{Der}(\mathfrak{g}, \mathfrak{m}),\{\cdot, \cdot\})$ is a strict Lie 2-algebra.

Proof. By Lemma 4.1 and Lemma 4.3, we only need to verify that $-\mathrm{D}$ is a graded derivation with respect to the bracket operation $\{\cdot, \cdot\}$, i.e., for any $X+l_{X} \in \operatorname{Der}_{0}(\mathfrak{g}, \mathfrak{m}), \Theta, \Theta^{\prime} \in \operatorname{Der}_{1}(\mathfrak{g}, \mathfrak{m})$,

$$
\begin{aligned}
-\mathrm{D}\left\{X+l_{X}, \Theta\right\} & =\left\{X+l_{X},-\mathrm{D} \Theta\right\} \\
\left\{-\mathrm{D} \Theta, \Theta^{\prime}\right\} & =\left\{\Theta,-\mathrm{D}^{\prime}\right\} .
\end{aligned}
$$

The left hand side of (22) is equal to $\delta\left([X, \Theta]_{\varphi}\right)+l_{\delta\left([X, \Theta]_{\varphi}\right)}$. By repeatedly applying condition $(i)$ 
of Definition 3.2 and the fact that $\Pi$ is a homomorphism, we have

$$
\begin{aligned}
& l_{\delta\left([X, \Theta]_{\varphi}\right)}(x, y) \\
= & {[X, \Theta]_{\varphi}[x, y]-x \triangleright[X, \Theta]_{\varphi} y+y \triangleright[X, \Theta]_{\varphi} x } \\
= & X \varphi\left(x \triangleright \Theta y-y \triangleright \Theta x+l_{\delta(\Theta)}(x, y)\right)-\Theta \varphi\left(x \triangleright X y-y \triangleright X x+\tilde{d} l_{X}(x, y)\right) \\
& -x \triangleright(X \varphi \Theta y-\Theta \varphi X y)+y \triangleright(X \varphi \Theta x-\Theta \varphi X x) \\
= & \left.X([x, \varphi \Theta y]+\sigma(x, \tilde{d} \Theta y)-[y, \varphi \Theta x]-\sigma(y, \tilde{d} \Theta x))+\varphi l_{\delta(\Theta)}(x, y)\right) \\
& -\Theta\left([x, \varphi X y]+d \sigma(x, X y)-[y, \varphi X x]-d \sigma(y, X x)+\varphi \tilde{d} l_{X}(x, y)\right) \\
& -x \triangleright(X \varphi \Theta y-\Theta \varphi X y)+y \triangleright(X \varphi \Theta x-\Theta \varphi X x) \\
= & x \triangleright X \varphi \Theta y-\varphi \Theta y \triangleright X x+l_{X}(x, d \varphi \Theta y)+X \sigma(x, \tilde{d} \Theta y) \\
& -y \triangleright X \varphi \Theta x+\varphi \Theta x \triangleright X y-l_{X}(y, d \varphi \Theta x)-X \sigma(y, \tilde{d} \Theta x)+X \varphi l_{\delta(\Theta)}(x, y) \\
& -x \triangleright \Theta \varphi X y+\varphi X y \triangleright \Theta x-l_{\delta(\Theta)}(x, \varphi X y)-\Theta d \sigma(x, X y) \\
& +y \triangleright \Theta \varphi X x-\varphi X x \triangleright \Theta y+l_{\delta(\Theta)}(y, \varphi X x)+\Theta d \sigma(y, X x)-\Theta \varphi \tilde{d} l_{X}(x, y) \\
& -x \triangleright(X \varphi \Theta y-\Theta \varphi X y)+y \triangleright(X \varphi \Theta x-\Theta \varphi X x) \\
= & l_{X}(x, d \varphi \Theta y)+X \sigma(x, \tilde{d} \Theta y)-l_{X}(y, d \varphi \Theta x)-X \sigma(y, \tilde{d} \Theta x)+X \varphi l_{\delta(\Theta)}(x, y) \\
& -l_{\delta(\Theta)}(x, \varphi X y)-\Theta d \sigma(x, X y)+l_{\delta(\Theta)}(y, \varphi X x)+\Theta d \sigma(y, X x)-\Theta \varphi \tilde{d} l_{X}(x, y) \\
= & l_{[X, \delta(\Theta)]_{\varphi}(x, y) .}
\end{aligned}
$$

The right hand side of (22) is equal to

$$
\left\{X+l_{X}, \delta(\Theta)+l_{\delta(\Theta)}\right\}=[X, \delta(\Theta)]_{\varphi}+l_{[X, \delta(\Theta)]_{\varphi}} .
$$

Thus, (22) holds since $\delta\left([X, \Theta]_{\varphi}\right)=[X, \delta(\Theta)]_{\varphi}$.

The equation (23) is a consequence of $\left[\delta(\Theta), \Theta^{\prime}\right]_{\varphi}=\left[\Theta, \delta\left(\Theta^{\prime}\right)\right]_{\varphi}$. This finishes the proof.

Proposition 4.5. For a crossed module $(\mathfrak{m}, \mathfrak{g}, \phi, \varphi, \sigma)$, the 1 st cohomology group $\mathrm{H}^{1}(\mathfrak{g}, \mathfrak{m})=$ $\operatorname{Der}_{0}(\mathfrak{g}, \mathfrak{m}) / \operatorname{Inn}_{0}(\mathfrak{g}, \mathfrak{m})$ is a quotient Lie algebra.

Proof. We shall prove $\operatorname{Inn}_{0}(\mathfrak{g}, \mathfrak{m})$ is an ideal of $\operatorname{Der}_{0}(\mathfrak{g}, \mathfrak{m})$. Since (22) holds, it suffices to show

$$
\left\{X+l_{X},-\mathrm{D} \alpha\right\}=-\mathrm{D}\left(X \varphi \alpha+l_{X}(\varphi \alpha, \cdot)+X \sigma(\alpha, \cdot)\right), \quad \forall \alpha \in \mathfrak{m}_{0} .
$$

Acting on $x \in \mathfrak{g}_{0}$ and taking into account (8) and (9), we have

$$
\begin{aligned}
\left\{X+l_{X},-\mathrm{D} \alpha\right\}(x) & =-X \varphi(\mathrm{D} \alpha) x+(\mathrm{D} \alpha) \varphi X x \\
& =-X \varphi(x \triangleright \alpha)+\varphi X x \triangleright \alpha \\
& =-x \triangleright X \varphi \alpha+\varphi \alpha \triangleright X x-\tilde{d} l_{X}(x, \varphi \alpha)-X d \sigma(x, \alpha)+\varphi X x \triangleright \alpha \\
& =-x \triangleright X \varphi \alpha-\tilde{d} l_{X}(x, \varphi \alpha)-\tilde{d} X \sigma(x, \alpha) \\
& =-\mathrm{D}\left(X \varphi \alpha+l_{X}(\varphi \alpha, \cdot)+X \sigma(\alpha, \cdot)\right)(x),
\end{aligned}
$$

where we have used condition $(i)$ and $\Pi$ is a homomorphism in Definition 3.2 Likewise, (24) holds on $\mathfrak{g}_{1}$. Finally, relying on the coherence law of the homomorphism $\Pi$, we have

$$
\begin{aligned}
& X\left(\sigma([x, y], \alpha)+\sigma(y \triangleright \alpha, x)-\sigma(x \triangleright \alpha, y)-\varphi((x, y) \triangleright \alpha)-l_{3}(x, y, \varphi \alpha)\right) \\
= & X([x, \sigma(y, \alpha)]+[y, \sigma(\alpha, x)]) \\
= & x \triangleright X \sigma(y, \alpha)-\sigma(y, \alpha) \triangleright X x+l_{X}(x, d \sigma(y, \alpha)) \\
& +y \triangleright X \sigma(\alpha, x)-\sigma(\alpha, x) \triangleright X y+l_{X}(y, d \sigma(\alpha, x)) .
\end{aligned}
$$


Thus, acting on $\wedge^{2} \mathfrak{g}_{0}$, it turns out that

$$
\begin{aligned}
& \left\{X+l_{X},-\mathrm{D} \alpha\right\}(x, y) \\
= & -X \varphi \mathrm{D} \alpha(x, y)+\mathrm{D} \alpha(\varphi X x, y)+\mathrm{D} \alpha(x, \varphi X y)+\mathrm{D} \alpha\left(\varphi l_{X}(x, y)\right)-l_{X}(\varphi \mathrm{D} \alpha(x), y) \\
& -l_{X}(x, \varphi \mathrm{D} \alpha(y))-X \sigma(\mathrm{D} \alpha(x), y)-X \sigma(x, \mathrm{D} \alpha(y))+\mathrm{D} \alpha(\sigma(X x, y))+\mathrm{D} \alpha(\sigma(x, X y)) \\
= & -X \varphi((x, y) \triangleright \alpha)+(\varphi X x, y) \triangleright \alpha+(x, \varphi X y) \triangleright \alpha+\varphi l_{X}(x, y) \triangleright \alpha-l_{X}([x, \varphi \alpha]+d \sigma(x, \alpha), y) \\
& -l_{X}(x,[y, \varphi \alpha]+d \sigma(y, \alpha))-X \sigma(x \triangleright \alpha, y)-X \sigma(x, y \triangleright \alpha)+\sigma(X x, y) \triangleright \alpha+\sigma(x, X y) \triangleright \alpha \\
= & \left(x \triangleright X \sigma(y, \alpha)-\sigma(y, \alpha) \triangleright X x+y \triangleright X \sigma(\alpha, x)-\sigma(\alpha, x) \triangleright X y-X \sigma([x, y], \alpha)+X l_{3}(x, y, \varphi \alpha)\right) \\
& +\left(l_{X}(\varphi \alpha,[x, y])-(x, y) \triangleright X \varphi \alpha-(y, \varphi \alpha) \triangleright X x-(\varphi \alpha, x) \triangleright X y+x \triangleright l_{X}(y, \varphi \alpha)\right. \\
& \left.+y \triangleright l_{X}(\varphi \alpha, x)-X l_{3}(x, y, \varphi \alpha)\right)+l_{\phi_{0}(y)}(X x, \alpha)-l_{\phi_{0}(x)}(X y, \alpha) \quad b y(25) \text { and (7) } \\
= & x \triangleright X \sigma(y, \alpha)+y \triangleright X \sigma(\alpha, x)-X \sigma([x, y], \alpha)+l_{X}(\varphi \alpha,[x, y])+x \triangleright l_{X}(y, \varphi \alpha)+y \triangleright l_{X}(\varphi \alpha, x) \\
& -(x, y) \triangleright X \varphi \alpha+\left(l_{\phi_{0}(y)}(\alpha, X x)-l_{\phi_{0}(x)}(\alpha, X y)+l_{\phi_{0}(y)}(X x, \alpha)-l_{\phi_{0}(x)}(X y, \alpha)\right) \\
= & -\mathrm{D}\left(X \varphi \alpha+l_{X}(\varphi \alpha, \cdot)+X \sigma(\alpha, \cdot)\right)(x, y),
\end{aligned}
$$

where we have used (iii) of Definition 3.2. The proof is finished.

Remark 4.6. Let $\mathfrak{m}=\mathfrak{g}, i=I d$ in Example 3.9. Then Theorem 4.4 recovers the theorem that $\operatorname{Der}(\mathfrak{g})$ is a strict Lie 2-algebra in [5] and Proposition 4.5 implies that $\mathrm{H}^{1}(\mathfrak{g})=\operatorname{Der}_{0}(\mathfrak{g}) / \operatorname{Inn}_{0}(\mathfrak{g})$ is a quotient Lie algebra. Also, it justifies our definition of $\operatorname{Inn}_{0}(\mathfrak{g})$, while it is defined by $\operatorname{Im}\left(\left.\mathrm{D}\right|_{\mathfrak{g}_{0}}\right)$ in [5] which is not an ideal of $\operatorname{Der}_{0}(\mathfrak{g})$ by 24).

Remark 4.7. It should be interesting to consider the strict 2-group integrated from the strict Lie 2-algebra $\operatorname{Der}(\mathfrak{g}, \mathfrak{m})$. In particular, for $\operatorname{Der}(\mathfrak{g})$, we can explicate the strict 2-group $\operatorname{Aut}(\mathfrak{g})$ consisted of all the automorphisms of Lie 2-algebra $\mathfrak{g}$. Furthermore, for a strict Lie 2-algebra $\mathfrak{g}$ with corresponding strict Lie 2 -groups $\mathcal{G}$, it is interesting to establish the connection between $\operatorname{Der}(\mathfrak{g})$ and the automorphism 2-group $\operatorname{Aut}(\mathcal{G})([21])$.

\section{Classification of strong crossed modules via $\mathrm{H}^{3}$}

\subsection{Crossed modules from extensions of short exact sequences}

Suppose $\left(\mathfrak{g}, d,[\cdot, \cdot], l_{3}\right)$ is a Lie 2-algebra and $\mathfrak{k} \subset \mathfrak{g}$ is an ideal. This gives rise to a short exact sequence of Lie 2-algebras

$$
0 \rightarrow \mathfrak{k} \hookrightarrow \mathfrak{g} \stackrel{\pi}{\longrightarrow} \mathfrak{h} \rightarrow 0,
$$

where $\mathfrak{h}=\mathfrak{g} / \mathfrak{k}$ and $\pi$ is the canonical projection, which is a strong Lie 2-algebra homomorphism. Furthermore, given an $\mathfrak{h}$-module $\mathbb{V}$, it is natural that $\mathbb{V}$ endows with a $\mathfrak{g}$-module structure and then a trivial $\mathfrak{h}$-module structure as follows:

$$
x \triangleright u=\tilde{x} \triangleright u, \quad(x, y) \triangleright u=(\tilde{x}, \tilde{y}) \triangleright u, \quad \forall x, y \in \mathfrak{g}, u \in \mathbb{V}, \tilde{x}=\pi(x), \tilde{y}=\pi(y) .
$$

The natural projection $\pi: \mathfrak{g} \longrightarrow \mathfrak{h}$ induces a map of cochain complexes:

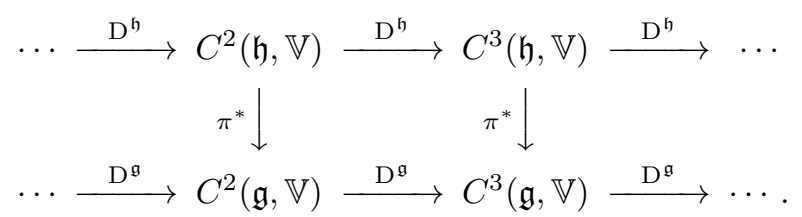

In this section, we always suppose $\alpha, \beta, \gamma \in \mathfrak{k}_{0}, \xi \in \mathfrak{k}_{1}, u, v, w \in V_{0}, m \in V_{1}$, and $x, y \in \mathfrak{g}_{0}, a \in \mathfrak{g}_{1}$. 
Lemma 5.1. For a 2-cochain $\lambda \in C^{2}(\mathfrak{g}, \mathbb{V})$, the following three statements are equivalent:

(1) $i_{e}\left(\mathrm{D}^{\mathfrak{g}} \lambda\right)=0, \forall e \in \mathfrak{k}$;

(2) $\mathrm{D}^{\mathfrak{g}} \lambda=\pi^{*} \theta$ for a 3 -cocycle $\theta \in C^{3}(\mathfrak{h}, \mathbb{V})$;

(3) The map $\phi^{\lambda}=\left(\phi_{0}^{\lambda}, \phi_{1}^{\lambda}, \phi_{2}^{\lambda}\right): \mathfrak{g} \rightarrow \operatorname{End}\left(\mathfrak{k} \oplus_{\lambda} \mathbb{V}\right)$ given below defines an action of $\mathfrak{g}$ on $\mathfrak{k} \oplus_{\lambda} \mathbb{V}$, where

$$
\mathfrak{k} \oplus_{\lambda} \mathbb{V}: \mathfrak{k}_{1} \oplus V_{1} \stackrel{d^{\lambda}}{\longrightarrow} \mathfrak{k}_{0} \oplus V_{0}
$$

is a 2-vector space with $d^{\lambda}(\xi+m)=d \xi+d^{\mathbb{V}} m+\lambda_{0}(\xi)$ and

$$
\left\{\begin{aligned}
x \triangleright_{\lambda}(\alpha+u) & =[x, \alpha]+\lambda_{1}(x, \alpha)+\tilde{x} \triangleright u \\
x \triangleright_{\lambda}(\xi+m) & =[x, \xi]+\lambda_{2}(x, \xi)+\tilde{x} \triangleright m, \\
a \triangleright_{\lambda}(\alpha+u) & =[a, \alpha]+\lambda_{2}(a, \alpha)+\tilde{a} \triangleright u \\
(x, y) \triangleright_{\lambda}(\alpha+u) & =-l_{3}(x, y, \alpha)-\lambda_{3}(x, y, \alpha)+(\tilde{x}, \tilde{y}) \triangleright u .
\end{aligned}\right.
$$

That is, $\phi^{\lambda}$ is a Lie 2-algebra homomorphism.

Proof. It is obvious that $(1) \Leftrightarrow(2)$, so it suffices to prove $(1) \Leftrightarrow(3)$. Suppose that $\lambda$ satisfies (1), we shall show that $\phi_{0}^{\lambda}(x) \in \operatorname{End}_{0}\left(\mathfrak{k} \oplus_{\lambda} \mathbb{V}\right)$ and $\phi^{\lambda}$ is a Lie 2-algebra homomorphism. Referring to (10), we use subscripts to distinguish the five components in $C^{3}(\mathfrak{g}, \mathbb{V})$.

Firstly, the equality $\phi_{0}^{\lambda}(x) \circ d^{\lambda}=d^{\lambda} \circ \phi_{0}^{\lambda}(x)$ holds since

$$
\begin{aligned}
& \left(\phi_{0}^{\lambda}(x) \circ d^{\lambda}-d^{\lambda} \circ \phi_{0}^{\lambda}(x)\right)(\xi+m) \\
= & x \triangleright_{\lambda}\left(d \xi+d^{\mathbb{V}} m+\lambda_{0}(\xi)\right)-d^{\lambda}\left([x, \xi]+\lambda_{2}(x, \xi)+\tilde{x} \triangleright m\right) \\
= & {[x, d \xi]+\lambda_{1}(x, d \xi)+\tilde{x} \triangleright\left(d^{\mathbb{V}} m+\lambda_{0}(\xi)\right)-d[x, \xi]-d^{\mathbb{V}} \lambda_{2}(x, \xi)-d^{\mathbb{V}}(\tilde{x} \triangleright m)-\lambda_{0}[x, \xi] } \\
= & \lambda_{1}(x, d \xi)+\tilde{x} \triangleright \lambda_{0}(\xi)-d^{\mathbb{V}} \lambda_{2}(x, \xi)-\lambda_{0}[x, \xi] \\
= & \left(D^{\mathfrak{g}} \lambda\right)_{0}(x, \xi) \\
= & 0
\end{aligned}
$$

where we have used the fact that $\triangleright$ is an action. Analogously, we get

$$
\begin{aligned}
\left(\phi_{0}^{\lambda}(d a)-\delta \phi_{1}^{\lambda}(a)\right)(\alpha+u) & =-\left(\mathrm{D}^{\mathfrak{g}} \lambda\right)_{0}(\alpha, a)=0, \\
\left(\phi_{0}^{\lambda}(d a)-\delta \phi_{1}^{\lambda}(a)\right)(\xi+m) & =-\left(\mathrm{D}^{\mathfrak{g}} \lambda\right)_{1}(a, \xi)=0 .
\end{aligned}
$$

Secondly, by an elementary computation, we obtain

$$
\begin{aligned}
& \left(\phi_{0}^{\lambda}[x, y]-\left[\phi_{0}^{\lambda}(x), \phi_{0}^{\lambda}(y)\right]-\delta \phi_{2}^{\lambda}(x, y)\right)(\alpha+u) \\
= & {[[x, y], \alpha]+\lambda_{1}([x, y], \alpha)+\widetilde{[x, y]} \triangleright u-[x,[y, \alpha]]-\lambda_{1}(x,[y, \alpha])-\tilde{x} \triangleright\left(\lambda_{1}(y, \alpha)+\tilde{y} \triangleright u\right) } \\
& +[y,[x, \alpha]]+\lambda_{1}(y,[x, \alpha])+\tilde{y} \triangleright\left(\lambda_{1}(x, \alpha)+\tilde{x} \triangleright u\right)+d l_{3}(x, y, \alpha) \\
& +d^{\mathbb{V}} \lambda_{3}(x, y, \alpha)-d^{\mathbb{V}}((\tilde{x}, \tilde{y}) \triangleright u)+\lambda_{0} l_{3}(x, y, \alpha) \\
= & \lambda_{1}([x, y], \alpha)+c \cdot p .-\tilde{x} \triangleright \lambda_{1}(y, \alpha)+\tilde{y} \triangleright \lambda_{1}(x, \alpha)+d^{\mathbb{V}} \lambda_{3}(x, y, \alpha)+\lambda_{0} l_{3}(x, y, \alpha) \\
= & -\left(D^{\mathfrak{g}} \lambda\right)_{2}(x, y, \alpha) \\
= & 0
\end{aligned}
$$

where we have used the general Jacobi identity of $\mathfrak{g}$ and the fact that $\triangleright$ is an action. Likewise, we can deduce that

$$
\begin{aligned}
\left(\phi_{0}^{\lambda}[x, y]-\left[\phi_{0}^{\lambda}(x), \phi_{0}^{\lambda}(y)\right]-\delta \phi_{2}^{\lambda}(x, y)\right)(\xi+m) & =-\left(\mathrm{D}^{\mathfrak{g}} \lambda\right)_{3}(x, y, \xi)=0 \\
\left(\phi_{1}^{\lambda}[x, a]-\left[\phi_{0}^{\lambda}(x), \phi_{1}^{\lambda}(a)\right]-\delta \phi_{2}^{\lambda}(x, d a)\right)(\alpha+u) & =-\left(\mathrm{D}^{\mathfrak{g}} \lambda\right)_{3}(x, a, \alpha)=0 .
\end{aligned}
$$


Finally, for the coherence condition, following from the fact that $\triangleright$ is an action and the coherence law of $[\cdot, \cdot]$ and $l_{3}$, we have

$$
\begin{aligned}
& \left(\left[\phi_{0}^{\lambda}(x), \phi_{2}^{\lambda}(y, z)\right]-\phi_{2}^{\lambda}([x, y], z)+c \cdot p .-\phi_{1}^{\lambda} l_{3}(x, y, z)\right)(\alpha+u) \\
=\quad & -\left[x, l_{3}(y, z, \alpha)\right]-\lambda_{2}\left(x, l_{3}(y, z, \alpha)\right)-\tilde{x} \triangleright \lambda_{3}(y, z, \alpha)+\tilde{x} \triangleright((\tilde{y}, \tilde{z}) \triangleright u) \\
& +l_{3}(y, z,[x, \alpha])+\lambda_{3}(y, z,[x, \alpha])-(\tilde{y}, \tilde{z}) \triangleright \lambda_{1}(x, \alpha)-(\tilde{y}, \tilde{z}) \triangleright(\tilde{x} \triangleright u) \\
& +l_{3}([x, y], z, \alpha)+\lambda_{3}([x, y], z, \alpha)-(\widetilde{[x, y]}, \tilde{z}) \triangleright u+c \cdot p \cdot(x, y, z) \\
& -\left[l_{3}(x, y, z), \alpha\right]-\lambda_{2}\left(l_{3}(x, y, z), \alpha\right)-l_{3}(x, y, z) \triangleright u \\
= & -\left(\mathrm{D}^{\mathfrak{g}} \lambda\right)_{4}(x, y, z, \alpha) \\
= & 0 .
\end{aligned}
$$

Thus $\phi^{\lambda}$ is a Lie 2-algebra homomorphism. From the process above, the other hand is obvious.

Define $\varphi: \mathfrak{k} \oplus_{\lambda} \mathbb{V} \longrightarrow \mathfrak{g}$ by $\varphi=i \oplus 0$, which is a chain map. Assume that $\lambda$ satisfies the condition $i_{e}\left(\mathrm{D}^{\mathfrak{g}} \lambda\right)=0, \forall e \in \mathfrak{k}$. Then by a simple check, we find that $\left(\mathfrak{k} \oplus_{\lambda} \mathbb{V}, \mathfrak{g}, \phi^{\lambda}, \varphi\right)$ satisfies all the conditions in Proposition 3.4. Thus, we obtain:

Proposition 5.2. Suppose that $i_{e}\left(\mathrm{D}^{\mathfrak{g}} \lambda\right)=0, \forall e \in \mathfrak{k}$. Then $\varepsilon_{\lambda}=\left(\mathfrak{k} \oplus_{\lambda} \mathbb{V}, \mathfrak{g}, \phi^{\lambda}, \varphi\right)$ is a strong crossed module, where the Lie 2-algebra structure on $\mathfrak{k} \oplus_{\lambda} \mathbb{V}$ is given by

$$
\left\{\begin{aligned}
{[\alpha+u, \beta+v]_{\lambda} } & =[\alpha, \beta]+\lambda_{1}(\alpha, \beta), \\
{[\alpha+u, \xi+m]_{\lambda} } & =[\alpha, \xi]+\lambda_{2}(\alpha, \xi) \\
l_{3}^{\lambda}(\alpha+u, \beta+v, \gamma+w) & =l_{3}(\alpha, \beta, \gamma)+\lambda_{3}(\alpha, \beta, \gamma)
\end{aligned}\right.
$$

and $l_{\phi_{0}^{\lambda}(x)}$ is defined by

$$
l_{\phi_{0}^{\lambda}(x)}(\alpha+u, \beta+v)=l_{3}(x, \alpha, \beta)+\lambda_{3}(x, \alpha, \beta) .
$$

Next, we deal with the problem of deformation. As will seen in the next proposition, we get the same strong crossed module in the isomorphic sense if $\lambda$ is modified by a coboundary and an element in $\operatorname{Im} \pi^{*}$.

Suppose $\lambda \in C^{2}(\mathfrak{g}, \mathbb{V})$ satisfying that $\mathrm{D}^{\mathfrak{g}} \lambda=\pi^{*} \theta$ for a 3 -cocycle $\theta \in C^{3}(\mathfrak{h}, \mathbb{V})$. Then for any $R \in C^{2}(\mathfrak{h}, \mathbb{V})$ and $A \in C^{1}(\mathfrak{g}, \mathbb{V})$, we have

$$
\mathrm{D}^{\mathfrak{g}}\left(\lambda+\mathrm{D}^{\mathfrak{g}} A+\pi^{*} R\right)=\pi^{*}\left(\theta+\mathrm{D}^{\mathfrak{h}} R\right)
$$

Note that $\varepsilon_{\lambda+D^{\mathfrak{g}} A+\pi^{*} R}=\varepsilon_{\lambda+\mathrm{D}^{\mathfrak{g}} A}$ due to $\left.\pi^{*} R\right|_{\mathfrak{k}}=0$. Then define $F: \mathfrak{k} \oplus_{\lambda+\mathrm{D}^{\mathfrak{g}} A} \mathbb{V} \longrightarrow \mathfrak{k} \oplus_{\lambda} \mathbb{V}$ by

$$
\left\{\begin{aligned}
F_{0}(\alpha+u) & =\alpha+u+A_{0}(\alpha), \\
F_{1}(\xi+m) & =\xi+m+A_{1}(\xi), \\
F_{2}(\alpha+u, \beta+v) & =A_{2}(\alpha, \beta)
\end{aligned}\right.
$$

$G=I d: \mathfrak{g} \longrightarrow \mathfrak{g}$, and $\tau: \mathfrak{g}_{0} \wedge\left(\mathfrak{k} \oplus_{\lambda+\mathrm{D}^{\mathfrak{g}} A} \mathbb{V}\right)_{0} \longrightarrow\left(\mathfrak{k} \oplus_{\lambda} \mathbb{V}\right)_{1}$ by $\tau(x, \alpha+u)=A_{2}(x, \alpha)$.

Proposition 5.3. The map $(F, G, \tau)$ is an isomorphism from $\varepsilon_{\lambda+\mathrm{D}^{\mathfrak{g}} A+\pi^{*} R}$ to $\varepsilon_{\lambda}$. We call it a gauge transformation.

Proof. Firstly, $F$ is a Lie 2-algebra homomorphism due to the formulation of $\mathrm{D}^{\mathfrak{g}} A$. Indeed, the condition $F_{0} \circ d^{\lambda+D^{\mathfrak{g}} A}=d^{\lambda} \circ F_{1}$ follows from

$$
\begin{aligned}
& \left(F_{0} \circ d^{\lambda+\mathrm{D}^{\mathfrak{g}} A}-d^{\lambda} \circ F_{1}\right)(\xi+m) \\
= & F_{0}\left(d \xi+d^{\mathbb{V}} m+\left(\lambda_{0}+\left(\mathrm{D}^{\mathfrak{g}} A\right)_{0}\right)(\xi)\right)-d^{\lambda}\left(\xi+m+A_{1}(\xi)\right) \\
= & d \xi+d^{\mathbb{V}} m+\lambda_{0}(\xi)+\left(\mathrm{D}^{\mathfrak{g}} A\right)_{0}(\xi)+A_{0}(d \xi)-d \xi-d^{\mathbb{V}}\left(m+A_{1}(\xi)\right)-\lambda_{0}(\xi) \\
= & \left(\mathrm{D}^{\mathfrak{g}} A\right)_{0}(\xi)+A_{0}(d \xi)-d^{\mathbb{V}} A_{1}(\xi) \\
= & 0 .
\end{aligned}
$$


Then, we shall verify the coherence condition, since the other two conditions of homomorphism are similar to get. By a straightforward calculation, we have

$$
\begin{aligned}
& {\left[F_{0}(\alpha+u), F_{2}(\beta+v, \gamma+w)\right]_{\lambda}-F_{2}\left([\alpha+u, \beta+v]_{\lambda+\mathrm{D}^{\mathfrak{g}} A}, \gamma+w\right)+c . p . } \\
& +l_{3}^{\lambda}\left(F_{0}(\alpha+u), F_{0}(\beta+v), F_{0}(\gamma+w)\right)-F_{1} l_{3}^{\lambda+D^{\mathfrak{g}} A}(\alpha+u, \beta+v, \gamma+w) \\
= & -A_{2}([\alpha, \beta], \gamma)+c . p .+l_{3}(\alpha, \beta, \gamma)-l_{3}(\alpha, \beta, \gamma)-\left(\mathrm{D}^{\mathfrak{g}} A\right)_{3}(\alpha, \beta, \gamma)-A_{1} l_{3}(\alpha, \beta, \gamma) \\
= & 0 .
\end{aligned}
$$

Next, we get $\varphi \circ F=G \circ \varphi$ by definition. Finally, similar to the above procedure, it is direct to check that

$$
\left(\left(G_{0}, F_{0}\right),\left(G_{1}, F_{1}\right),\left(G_{2}, \tau, F_{2}\right)\right): \mathfrak{g} \triangleright_{\lambda+\mathrm{D}^{\mathfrak{g}} A}\left(\mathfrak{k} \oplus_{\lambda+\mathrm{Dg}_{A}} \mathbb{V}\right) \longrightarrow \mathfrak{g} \triangleright_{\lambda}\left(\mathfrak{k} \oplus_{\lambda} \mathbb{V}\right)
$$

is a Lie 2-algebra homomorphism. Thus, $(F, G, \tau)$ is a morphism from $\varepsilon_{\lambda+\mathrm{D}^{\mathfrak{g}} A}$ to $\varepsilon_{\lambda}$. Furthermore, note that $F$ and $G$ are bijections as chain maps and $\varepsilon_{\lambda+D^{\mathfrak{g}} A+\pi^{*} R}=\varepsilon_{\lambda+D^{\mathfrak{g}} A}$. We conclude that $\varepsilon_{\lambda+D^{\mathfrak{g}} A+\pi^{*} R}$ and $\varepsilon_{\lambda}$ are isomorphic.

Generally, for a 3-cocycle $\theta \in C^{3}(\mathfrak{h}, \mathbb{V})$, the 3 -cochain $\pi^{*} \theta$ is not necessarily to be a 3 coboundary. Nevertheless, consider the short exact sequence

$$
0 \rightarrow \operatorname{ker} \pi \hookrightarrow \mathfrak{F}(\mathfrak{h}) \stackrel{\pi}{\longrightarrow} \mathfrak{h} \rightarrow 0,
$$

where $\mathfrak{F}(\mathfrak{h})$ is the free Lie 2-algebra ([17) generated by the underlying 2-vector space of $\mathfrak{h}$ and $\pi$ is the canonical projection. For a 3-cocycle $\theta \in C^{3}(\mathfrak{h}, \mathbb{V})$, since the second and third cohomology of any free Lie 2-algebra are trivia $\sqrt{1}$, there exists a 2-cochain $\lambda \in C^{2}(\mathfrak{F}(\mathfrak{h}), \mathbb{V})$ such that $\mathrm{D}^{\mathfrak{F}(\mathfrak{h})} \lambda=\pi^{*} \theta$ and for different 2-cochains $\lambda, \lambda^{\prime}$ satisfying it, we have $\left[\lambda-\lambda^{\prime}\right]=0$. By Proposition [5.3, we have:

Corollary 5.4. For any $[\theta] \in \mathrm{H}^{3}(\mathfrak{h}, \mathbb{V})$, we get a class of crossed modules differing from each other by a gauge transformation

$$
\left\{\varepsilon_{\lambda} ; D^{\widetilde{F}(\mathfrak{h})} \lambda=\pi^{*} \theta^{\prime},\left[\theta^{\prime}\right]=[\theta] \in \mathrm{H}^{3}(\mathfrak{h}, \mathbb{V})\right\} .
$$

\subsection{Classification}

A crossed module $(\mathfrak{m}, \mathfrak{g}, \phi, \varphi, \sigma)$ can yield a 4 -term exact sequence of 2-vector spaces

$$
0 \longrightarrow \mathbb{V} \stackrel{i}{\longrightarrow} \mathfrak{m} \stackrel{\varphi}{\longrightarrow} \mathfrak{g} \stackrel{\pi}{\longrightarrow} \mathfrak{h} \longrightarrow 0
$$

where $\mathbb{V} \triangleq \operatorname{ker} \varphi, \mathfrak{h} \triangleq \operatorname{coker} \varphi$, and $i, \pi$ are the canonical inclusion and projection. By $(i),(i i)$ of Definition 3.2 $\mathbb{V}$ is in the center of $\mathfrak{m}$. However, it needs some extra conditions to ensure that $\mathfrak{h}$ is a Lie 2-algebra and there exists an induced action of $\mathfrak{h}$ on $\mathbb{V}$.

Lemma 5.5. For a crossed module of Lie 2-algebras $(\mathfrak{m}, \mathfrak{g}, \phi, \varphi, \sigma)$, we have

(1) $\mathfrak{h}=\mathfrak{g} / \operatorname{Im} \varphi$ is a quotient Lie 2-algebra of $\mathfrak{g}$ if $\operatorname{Im} \sigma \subset \operatorname{Im} \varphi_{1}$;

(2) the action of $\mathfrak{g}$ on $\mathfrak{m}$ induces an $\mathfrak{h}$-module structure on $\mathbb{V}$ if $\operatorname{Im} \sigma \subset \operatorname{Im} \varphi_{1}$ and $\sigma\left(\operatorname{ker} \varphi_{0} \wedge \mathfrak{g}_{0}\right)=0$.

In particular, $\mathbb{V}$ becomes a module of Lie 2-algebra $\mathfrak{h}$ if the crossed module is strong.

\footnotetext{
${ }^{1}$ It is due to that the cohomology of Lie 2-algebra is "operadic" in nature and the operadic cohomology (degree $\geq 2$ ) vanishes on frees. See [18.
} 
Proof. In order to prove $\operatorname{Im} \varphi$ is an ideal, we need to verify $l_{2}(\operatorname{Im} \varphi \wedge \mathfrak{g}) \subset \operatorname{Im} \varphi, l_{3}\left(\operatorname{Im} \varphi_{0} \wedge \mathfrak{g}_{0} \wedge \mathfrak{g}_{0}\right) \subset$ $\operatorname{Im} \varphi_{1}$. Following that $\Pi=I d+\sigma+\varphi$ is a Lie 2 -algebra homomorphism, we have

$$
\left\{\begin{array}{rlrl}
\varphi_{0}(x \triangleright \alpha)-\left[x, \varphi_{0}(\alpha)\right] & =d \sigma(x, \alpha), & & \forall x \in \mathfrak{g}_{0}, \alpha \in \mathfrak{m}_{0}, \\
\varphi_{1}(a \triangleright \alpha)-\left[a, \varphi_{0}(\alpha)\right] & =\sigma(d a, \alpha), & \forall a \in \mathfrak{g}_{1}, \alpha \in \mathfrak{m}_{0}, \\
\varphi_{1}(x \triangleright \xi)-\left[x, \varphi_{1}(\xi)\right] & =\sigma(x, \tilde{d} \xi), & & \forall x \in \mathfrak{g}_{0}, \xi \in \mathfrak{m}_{1},
\end{array}\right.
$$

which leads to $l_{2}(\operatorname{Im} \varphi \wedge \mathfrak{g}) \subset \operatorname{Im} \varphi$ since $\operatorname{Im} \sigma \subset \operatorname{Im} \varphi_{1}$. Moreover, consider the coherence law in the definition of homomorphism, for any $x, y \in \mathfrak{g}_{0}, \alpha \in \mathfrak{m}_{0}$,

$$
[\sigma(y, \alpha), x]+[\sigma(\alpha, x), y]+\sigma([x, y], \alpha)+\sigma(y \triangleright \alpha, x)-\sigma(x \triangleright \alpha, y)=l_{3}\left(x, y, \varphi_{0} \alpha\right)-\varphi_{1} L_{3}(x, y, \alpha)
$$

which implies that $l_{3}\left(x, y, \varphi_{0} \alpha\right) \in \operatorname{Im} \varphi_{1}$, i.e., $l_{3}\left(\operatorname{Im} \varphi_{0} \wedge \mathfrak{g}_{0} \wedge \mathfrak{g}_{0}\right) \subset \operatorname{Im} \varphi_{1}$.

To prove (2), firstly, by equalities (30) and $\sigma\left(\operatorname{ker} \varphi_{0} \wedge \mathfrak{g}_{0}\right)=0$, we get $\phi_{i}: \mathfrak{g}_{\mathfrak{i}} \longrightarrow \operatorname{End}_{i}(\operatorname{ker} \varphi), i=$ 0,1 . Coupled with the coherence law above, it is clear that

$$
(x, y) \triangleright \beta=-L_{3}(x, y, \beta) \in \operatorname{ker} \varphi_{1}, \quad \forall \beta \in \operatorname{ker} \varphi_{0}, x, y \in \mathfrak{g}_{0} .
$$

Namely, $\phi_{2}: \wedge^{2} \mathfrak{g}_{0} \longrightarrow \operatorname{End}_{1}(\operatorname{ker} \varphi)$. Thus we can define $\tilde{\phi}=\phi \circ s: \mathfrak{h} \longrightarrow \operatorname{End}(\operatorname{ker} \varphi)$, where $s: \mathfrak{h} \longrightarrow \mathfrak{g}$ is a section of $\pi$.

Next, we prove $\tilde{\phi}$ is independent of the section $s$. Let $s^{\prime}$ be another section of $\pi$, then $\operatorname{Im}\left(s-s^{\prime}\right) \in$ $\operatorname{ker} \pi=\operatorname{Im} \varphi$. We shall check $\phi \circ\left(s-s^{\prime}\right)=0$, which is equivalent to show the homomorphism $\mathfrak{g} \stackrel{\phi}{\longrightarrow} \operatorname{End}(\operatorname{ker} \varphi)$ vanishes when restricting on $\operatorname{Im} \varphi$. Depending on $(i),($ iii $)$ of Definition 3.2 , we have

$$
\varphi(\alpha) \triangleright \beta=-\varphi(\beta) \triangleright \alpha=0, \quad \forall \beta \in \operatorname{ker} \varphi, \alpha \in \mathfrak{m} .
$$

And, for any $\beta \in \operatorname{ker} \varphi_{0}, \alpha \in \mathfrak{m}_{0}, x \in \mathfrak{g}_{0}$,

$$
\left(x, \varphi_{0} \alpha\right) \triangleright \beta=-\left(x, \varphi_{0} \beta\right) \triangleright \alpha-\sigma(x, \beta) \triangleright \alpha-\sigma(x, \alpha) \triangleright \beta=-\sigma(x, \alpha) \triangleright \beta,
$$

which vanishes since $\operatorname{Im} \sigma \subset \operatorname{Im} \varphi_{1}$ and $\beta \in \operatorname{ker} \varphi_{0}$.

At last, since $[s x, s y] \triangleright \alpha=s[x, y] \triangleright \alpha, \forall x, y \in \mathfrak{h}, \alpha \in \operatorname{ker} \varphi$, it is direct to check $\tilde{\phi}$ is a Lie 2-algebra homomorphism. Therefore, the requirements $\operatorname{Im} \sigma \subset \operatorname{Im} \varphi_{1}$ and $\sigma\left(\operatorname{ker} \varphi_{0} \wedge \mathfrak{g}_{0}\right)=0$ make sure that the action of $\mathfrak{g}$ on $\mathfrak{m}$ can induce an action of $\mathfrak{h}$ on $\operatorname{ker} \varphi$.

Note that for a strong crossed module $(\mathfrak{m}, \mathfrak{g}, \phi, \varphi)$, there exists an exact sequence (29) satisfying that all the homomorphisms are strong. Denote by $\mathcal{C}(\mathfrak{h}, \mathbb{V})$ the set of strong crossed modules with respect to fixed kernel $\mathbb{V}$ and fixed cokernel $\mathfrak{h}$ and fixed action of $\mathfrak{h}$ on $\mathbb{V}$.

Example 5.6. Consider the set in Proposition 5.4 denoted by $\mathcal{C}(\mathfrak{h}, \mathbb{V})_{[\theta]}$. In fact,

$$
\mathcal{C}(\mathfrak{h}, \mathbb{V})_{[\theta]}=\left\{\varepsilon_{\lambda} ; D^{\mathfrak{F}(\mathfrak{h})} \lambda=\pi^{*} \theta^{\prime},\left[\theta^{\prime}\right]=[\theta] \in \mathrm{H}^{3}(\mathfrak{h}, \mathbb{V})\right\}
$$

is a subset of $\mathcal{C}(\mathfrak{h}, \mathbb{V})$.

Definition 5.7. Let $(\mathfrak{m}, \mathfrak{g}, \phi, \varphi)$ and $\left(\mathfrak{m}^{\prime}, \mathfrak{g}^{\prime}, \phi^{\prime}, \varphi^{\prime}\right)$ be two strong crossed modules in $\mathcal{C}(\mathfrak{h}, \mathbb{V}), a$ strong map between them is a strong morphism of crossed modules $(F, G)$ such that the diagram

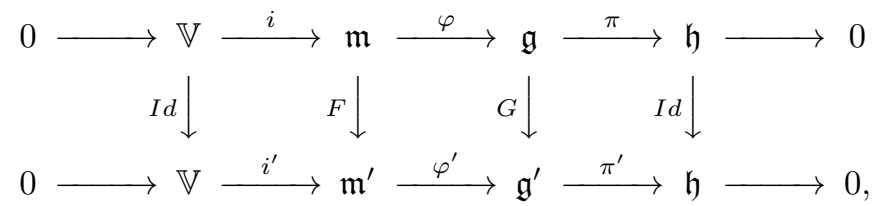

is commutative. 
Definition 5.8. For two crossed module $\varepsilon, \varepsilon^{\prime} \in \mathcal{C}(\mathfrak{h}, \mathbb{V})$, we define $\varepsilon \sim \varepsilon^{\prime}$ if there exist two crossed module $\varepsilon_{\lambda}, \varepsilon_{\lambda^{\prime}} \in \mathcal{C}(\mathfrak{h}, \mathbb{V})_{[\theta]}$ for a 3 -cocycle $\theta \in C^{3}(\mathfrak{h}, \mathbb{V})$ such that the diagram

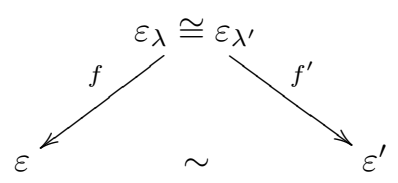

holds, where $f, f^{\prime}$ are strong maps in $\mathcal{C}(\mathfrak{h}, \mathbb{V})$ and $\cong$ is the gauge transformation between them.

Since the composition of gauge transformations is also a gauge transformation, it is evident that $\sim$ is an equivalence relation.

Remark 5.9. The linear map $\varphi: \mathfrak{m} \rightarrow \mathfrak{g}$ induces an action groupoid $\mathfrak{g} \times \mathfrak{m} \rightrightarrows \mathfrak{g}$ with the abelian group structure on $\mathfrak{m}$, where the source, target and inclusion maps are $s(x, \alpha)=x, t(x, \alpha)=$ $x+\varphi(\alpha), i(x)=(x, 0)$. Then $\varphi$ has fixed kernel and cokernel means that the groupoid has fixed isotropy subgroup and orbit space. In this viewpoint, the relation between $\varepsilon$ and $\varepsilon^{\prime}$ defined by (32) is in fact a generalized map ([19]]) between them.

Theorem 5.10. For a Lie 2-algebra $\mathfrak{h}$ and an $\mathfrak{h}$-module $\mathbb{V}$, there exists a canonical bijection

$$
\mathcal{C}(\mathfrak{h}, \mathbb{V}) / \sim \stackrel{\approx}{\longrightarrow} \mathrm{H}^{3}(\mathfrak{h}, \mathbb{V}),
$$

where $\sim$ is the equivalence relation defined in Definition 5.8 .

We divide the proof into four steps. For simplicity, we denote by the same notations $d,[\cdot, \cdot]$ and $l_{3}$ for different Lie 2-algebras except when emphasis is needed, which will not cause any confusion. In the rest of this section, we will always suppose $x, y, z \in \mathfrak{h}_{0}$ and $a, b \in \mathfrak{h}_{1}$.

Step 1: Construct a map $\mu: \mathcal{C}(\mathfrak{h}, \mathbb{V}) \longrightarrow H^{3}(\mathfrak{h}, \mathbb{V})$. To a crossed module $\varepsilon=(\mathfrak{m}, \mathfrak{g}, \phi, \varphi) \in$ $\mathcal{C}(\mathfrak{h}, \mathbb{V})$, choose linear sections $s=\left(s_{0}, s_{1}\right): \mathfrak{h} \rightarrow \mathfrak{g}, \pi s=I d$ and $q=\left(q_{0}, q_{1}\right): \operatorname{Im} \varphi \rightarrow \mathfrak{m}, \varphi q=I d$. Since $d s_{1}(a)-s_{0} d(a) \in \operatorname{ker} \pi_{0}=\operatorname{Im} \varphi_{0}$, we can take

$$
\lambda_{0}(a)=q_{0}\left(d s_{1}(a)-s_{0} d(a)\right) .
$$

Similarly, take

$$
\left\{\begin{aligned}
\lambda_{1}(x, y) & =q_{0}\left(\left[s_{0}(x), s_{0}(y)\right]-s_{0}[x, y]\right) \\
\lambda_{2}(x, a) & =q_{1}\left(\left[s_{0}(x), s_{1}(a)\right]-s_{1}[x, a]\right) \\
\lambda_{3}(x, y, z) & =q_{1}\left(l_{3}\left(s_{0}(x), s_{0}(y), s_{0}(z)\right)-s_{1} l_{3}(x, y, z)\right)
\end{aligned}\right.
$$

Note that $\lambda_{\varepsilon}=\sum_{i=0}^{3} \lambda_{i}$ satisfies $\pi^{*} \lambda_{\varepsilon} \in C^{2}(\mathfrak{g}, \mathfrak{m})$. It is reasonable to define

$$
\theta_{\varepsilon}=s^{*} \mathrm{D}^{\mathfrak{g}}\left(\pi^{*} \lambda_{\varepsilon}\right) \text {. }
$$

Lemma 5.11. With the above notations, we have

(1) $\varphi \circ \theta_{\varepsilon}=0$, that is, $\theta_{\varepsilon} \in C^{3}(\mathfrak{h}, \mathbb{V})$.

(2) $\mathrm{D}^{\mathfrak{h}} \theta_{\varepsilon}=0$.

Proof. According to (10), we shall check that $\varphi_{0} \circ \theta_{\varepsilon j}=0$ for $\mathrm{j}=0,2$ and $\varphi_{1} \circ \theta_{\varepsilon j}=0$ for $\mathrm{j}=1,3,4$. The cases of $j=0,1,2,3$ are quite straightforward, so we omit the details. For the case of $j=4$, since $\Pi=I d+\varphi$ is a strong homomorphism, we have

$$
l_{3}\left(s_{0}\left(x_{\sigma_{1}}\right), s_{0}\left(x_{\sigma_{2}}\right), \varphi_{0} \lambda_{1}\left(x_{\sigma_{3}}, x_{\sigma_{4}}\right)\right)=-\varphi_{1}\left(\left(s_{0}\left(x_{\sigma_{1}}\right), s_{0}\left(x_{\sigma_{2}}\right)\right) \triangleright \lambda_{1}\left(x_{\sigma_{3}}, x_{\sigma_{4}}\right)\right) .
$$


Taking into account (10), (34) and the coherence laws of $l_{2}, l_{3}$ of Lie 2-algebras $\mathfrak{g}$ and $\mathfrak{h}$, we have

$$
\begin{aligned}
& \varphi_{1}\left(\theta_{\varepsilon 4}\left(x_{1}, x_{2}, x_{3}, x_{4}\right)\right) \\
= & -\sum_{\sigma}(-1)^{\sigma} l_{3}\left(s_{0}\left(x_{\sigma_{1}}\right), s_{0}\left(x_{\sigma_{2}}\right),\left[s_{0}\left(x_{\sigma_{3}}\right), s_{0}\left(x_{\sigma_{4}}\right)\right]-s_{0}\left[x_{\sigma_{3}}, x_{\sigma_{4}}\right]\right) \\
& -\sum_{\tau}(-1)^{\tau}\left\{\left[s_{0}\left(x_{\tau_{4}}\right), s_{1} l_{3}\left(x_{\tau_{1}}, x_{\tau_{2}}, x_{\tau_{3}}\right)\right]-s_{1}\left[x_{\tau_{4}}, l_{3}\left(x_{\tau_{1}}, x_{\tau_{2}}, x_{\tau_{3}}\right)\right]\right\} \\
& +\sum_{i=1}^{4}(-1)^{i+1}\left[s_{0}\left(x_{i}\right), l_{3}\left(s_{0}\left(x_{1}\right), \cdots, s_{0}\left(\widehat{x_{i}}\right), \cdots, s_{0}\left(x_{4}\right)\right)-s_{1} l_{3}\left(x_{1}, \cdots, \widehat{x_{i}}, \cdots, x_{4}\right)\right] \\
& +\sum_{i<j}(-1)^{i+j}\left\{l_{3}\left(s_{0}\left[x_{i}, x_{j}\right], s_{0}\left(x_{1}\right), \cdots, s_{0}\left(\widehat{x_{i}}\right), \cdots, s_{0}\left(\widehat{x_{j}}\right), \cdots, s_{0}\left(x_{4}\right)\right)\right. \\
& \left.-s_{1} l_{3}\left(\left[x_{i}, x_{j}\right], x_{1}, \cdots, \widehat{x_{i}}, \cdots, \widehat{x_{j}}, \cdots, x_{4}\right)\right\} \\
= & -\sum_{\sigma}(-1)^{\sigma} l_{3}\left(s_{0}\left(x_{\sigma_{1}}\right), s_{0}\left(x_{\sigma_{2}}\right),\left[s_{0}\left(x_{\sigma_{3}}\right), s_{0}\left(x_{\sigma_{4}}\right)\right]\right) \\
& +\sum_{i=1}^{4}(-1)^{i+1}\left[s_{0}\left(x_{i}\right), l_{3}\left(s_{0}\left(x_{1}\right), \cdots, s_{0}\left(\widehat{x_{i}}\right), \cdots, s_{0}\left(x_{4}\right)\right)\right] \\
& +\sum_{\tau}(-1)^{\tau} s_{1}\left[x_{\tau_{4}}, l_{3}\left(x_{\tau_{1}}, x_{\tau_{2}}, x_{\tau_{3}}\right)\right]+\sum_{i<j}(-1)^{i+j} s_{1} l_{3}\left(\left[x_{i}, x_{j}\right], x_{1}, \cdots, \widehat{x_{i}}, \cdots, \widehat{x_{j}}, \cdots, x_{4}\right) \\
= & 0 .
\end{aligned}
$$

Therefore, $\operatorname{Im} \theta_{\varepsilon} \subset \mathbb{V}$.

Note that if only $\operatorname{Im} \lambda_{\varepsilon} \subset \mathbb{V}$, the definition of $\theta_{\varepsilon}$ would be read as $\theta_{\varepsilon}=s^{*} \pi^{*} D^{\mathfrak{h}}\left(\lambda_{\varepsilon}\right)=D^{\mathfrak{h}} \lambda_{\varepsilon}$ and hence would give $\mathrm{D}^{\mathfrak{h}} \theta_{\varepsilon}=0$. In our situation, straightforward calculations show that $\mathrm{D}^{\mathfrak{h}} \theta_{\varepsilon}$ still vanishes. Explicitly, by the definition of $\mathrm{D}^{\mathfrak{h}}$, we have

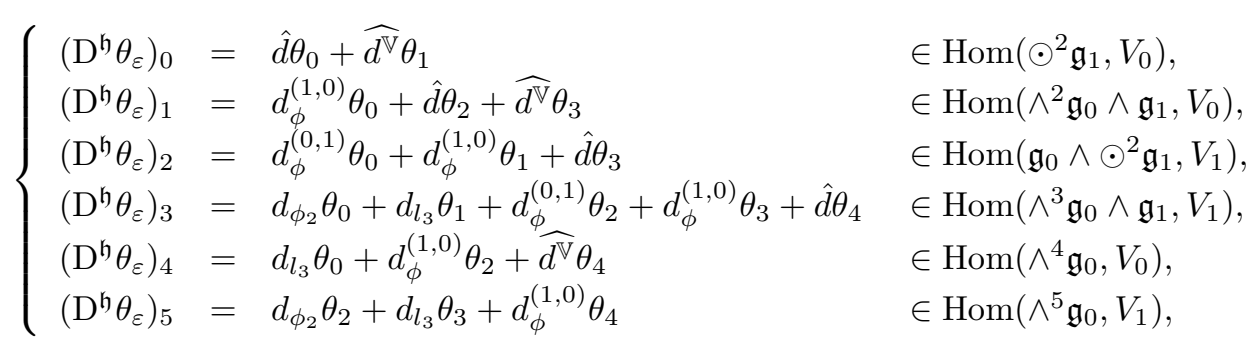

where $\theta_{j}=\theta_{\varepsilon j}=\left(s^{*} \mathrm{D}^{\mathfrak{g}}\left(\pi^{*} \lambda_{\varepsilon}\right)\right)_{j}$ as in (10). By direct calculations, we have

$$
\begin{aligned}
\left(D^{\mathfrak{h}} \theta_{\varepsilon}\right)_{0}(a, b)= & -s_{0}(d a) \triangleright \lambda_{0}(b)+\lambda_{0}[d a, b]-\lambda_{1}(d a, d b)+d^{\mathfrak{m}} \lambda_{2}(d a, b) \\
& -s_{0}(d b) \triangleright \lambda_{0}(a)+\lambda_{0}[d b, a]-\lambda_{1}(d b, d a)+d^{\mathfrak{m}} \lambda_{2}(d b, a) \\
& +d^{\mathbb{V}}\left(s_{1}(a) \triangleright \lambda_{0}(b)+s_{1}(b) \triangleright \lambda_{0}(a)-\lambda_{2}(d a, b)+\lambda_{2}(a, d b)\right) \\
= & \varphi_{0} \lambda_{0}(a) \triangleright \lambda_{0}(b)+\varphi_{0} \lambda_{0}(b) \triangleright \lambda_{0}(a),
\end{aligned}
$$

which vanishes due to condition $(i)$ of Definition 3.2. Similarly, we can deduce that $\mathrm{D}^{\mathfrak{h}} \theta_{\varepsilon}=0$.

As has been already demonstrated, to each strong crossed module $\varepsilon=(\mathfrak{m}, \mathfrak{g}, \phi, \varphi) \in \mathcal{C}(\mathfrak{h}, \mathbb{V})$, one can obtain a 3 -cocycle $\theta_{\varepsilon} \in C^{3}(\mathfrak{h}, \mathbb{V})$. Then, define $\mu(\varepsilon)=\left[\theta_{\varepsilon}\right]$.

Step 2: We shall prove the canonical property of the map $\mu$. Namely, $\mu$ is independent of the choices made of sections $s$ and $q$. Here, for future reference, we also prove that if there is a strong map $\varepsilon \rightarrow \varepsilon^{\prime}$ in $\mathcal{C}(\mathfrak{h}, V)$, then $\theta_{\varepsilon}$ equals $\theta_{\varepsilon^{\prime}}$ in $\mathrm{H}^{3}(\mathfrak{h}, \mathbb{V})$. 
Lemma 5.12. $\theta_{\varepsilon}$ is independent of the choice of section $s$.

Proof. Suppose that $\bar{s}=\left(\bar{s}_{0}, \bar{s}_{1}\right)$ is another section of $\pi$ and let $\bar{\theta}_{\varepsilon}$ be the 3-cocycle defined using $\bar{s}$ instead of $s$. We need to prove that $\bar{\theta}_{\varepsilon}$ coincides with $\theta_{\varepsilon}$ in $\mathrm{H}^{3}(\mathfrak{h}, \mathbb{V})$.

Since $\bar{s}$ and $s$ are both sections of $\pi$, there exist two linear maps $t_{i}: \mathfrak{h}_{i} \longrightarrow \mathfrak{m}_{i}$ with $s_{i}-\bar{s}_{i}=\varphi_{i} \circ t_{i}$. Construct four maps as follows, for any $x, y, z \in \mathfrak{h}_{0}, a \in \mathfrak{h}_{1}$,

$$
\left\{\begin{aligned}
B_{0}(a)= & d^{\mathfrak{m}} t_{1}(a)-t_{0}(d a) \\
B_{1}(x, y)= & \bar{s}_{0}(x) \triangleright t_{0}(y)-s_{0}(y) \triangleright t_{0}(x)-t_{0}[x, y], \\
B_{2}(x, a)= & \bar{s}_{0}(x) \triangleright t_{1}(a)-s_{1}(a) \triangleright t_{0}(x)-t_{1}[x, a] \\
B_{3}(x, y, z)= & -\left(s_{0}(x), s_{0}(y)\right) \triangleright t_{0}(z)-\left(\bar{s}_{0}(x), \bar{s}_{0}(y)\right) \triangleright t_{0}(z)+\left(s_{0}(x), \bar{s}_{0}(y)\right) \triangleright t_{0}(z) \\
& -\left(\bar{s}_{0}(y), s_{0}(z)\right) \triangleright t_{0}(x)-\left(\bar{s}_{0}(z), s_{0}(x)\right) \triangleright t_{0}(y) .
\end{aligned}\right.
$$

Since $\varphi_{0} d=d^{m} \varphi_{1}$, it is obvious that

$$
\begin{aligned}
\varphi_{0}\left(B_{0}(a)\right) & =d s_{1}(a)-d \bar{s}_{1}(a)-s_{0}(d a)+\bar{s}_{0}(d a) \\
& =\varphi_{0}\left(\lambda_{0}(a)-\bar{\lambda}_{0}(a)\right)
\end{aligned}
$$

which implies that

$$
\lambda_{0}-\bar{\lambda}_{0}-B_{0} \in \operatorname{Hom}\left(\mathfrak{h}_{1}, V_{0}\right) .
$$

Similarly, relying on $\Pi=I d+\varphi$ is a strong homomorphism, we can deduce that $\lambda-\bar{\lambda}-B \in C^{2}(\mathfrak{h}, \mathbb{V})$, where $B=\sum_{i=0}^{3} B_{i}$. Furthermore, we claim that

$$
\theta_{\varepsilon}-\bar{\theta}_{\varepsilon}=\mathrm{D}^{\mathfrak{h}}(\lambda-\bar{\lambda}-B) .
$$

By straightforward computations, we have

$$
\begin{aligned}
\left(\theta_{\varepsilon}-\bar{\theta}_{\varepsilon}\right)_{0}(x, a)= & s_{0}(x) \triangleright\left(\lambda_{0}-\bar{\lambda}_{0}\right)(a)-\left(\lambda_{0}-\bar{\lambda}_{0}\right)[x, a]+\varphi_{0} t_{0}(x) \triangleright \bar{\lambda}_{0}(a) \\
& +\left(\lambda_{1}-\bar{\lambda}_{1}\right)(x, d a)-d^{\mathfrak{m}}\left(\lambda_{2}-\bar{\lambda}_{2}\right)(x, a),
\end{aligned}
$$

and

$$
\begin{aligned}
\left(D^{\mathfrak{h}}(\lambda-\bar{\lambda}-B)\right)_{0}(x, a)= & s_{0}(x) \triangleright\left(\lambda_{0}-\bar{\lambda}_{0}-B_{0}\right)(a)-\left(\lambda_{0}-\bar{\lambda}_{0}-B_{0}\right)[x, a] \\
& +\left(\lambda_{1}-\bar{\lambda}_{1}-B_{1}\right)(x, d a)-d^{\mathfrak{m}}\left(\lambda_{2}-\bar{\lambda}_{2}-B_{2}\right)(x, a) .
\end{aligned}
$$

Then substituting $B_{i}$ by the right hand sides of (35) and taking into account condition $(i)$ of Definition 3.2, we get

$$
\begin{aligned}
& \\
= & s_{0}(x) \triangleright\left(d^{\mathfrak{m}} t_{1}(a)-t_{0}(d a)\right)-d^{\mathfrak{m}} t_{1}[x, a]+t_{0}[x, d a]+\left(\bar{s}_{0}(d a)-d \bar{s}_{1}(a)\right) \triangleright t_{0}(x) \\
& +\bar{s}_{0}(x) \triangleright t_{0}(d a)-s_{0}(d a) \triangleright t_{0}(x)-t_{0}[x, d a]-d^{\mathfrak{m}}\left(\bar{s}_{0}(x) \triangleright t_{1}(a)-s_{1}(a) \triangleright t_{0}(x)-t_{1}[x, a]\right) \\
= & \varphi_{0} t_{0}(x) \triangleright d^{\mathfrak{m}} t_{1}(a)+d^{\mathfrak{m}}\left(\varphi_{1} t_{1}(a) \triangleright t_{0}(x)\right)-\varphi_{0} t_{0}(x) \triangleright t_{0}(d a)-\varphi_{0} t_{0}(d a) \triangleright t_{0}(x) \\
= & 0 .
\end{aligned}
$$

Similarly, we can verify that $\theta_{\varepsilon}-\bar{\theta}_{\varepsilon}=\mathrm{D}^{\mathfrak{h}}(\lambda-\bar{\lambda}-B)$, which proves that the class of $\theta_{\varepsilon}$ is independent of the section $s$.

Lemma 5.13. $\theta_{\varepsilon}$ is independent of the choice of section $q$ and a strong map $\varepsilon \longrightarrow \varepsilon^{\prime}$. 
Proof. Consider a strong map $(F, G): \varepsilon \longrightarrow \varepsilon^{\prime}$ as in Definition [5.7. Let $s: \mathfrak{h} \longrightarrow \mathfrak{g}$ and $q: \operatorname{Im} \varphi \longrightarrow \mathfrak{m}$ be sections of $\pi$ and $\varphi$ and let $s^{\prime}: \mathfrak{h}^{\prime} \longrightarrow \mathfrak{g}^{\prime}$ and $q^{\prime}: \operatorname{Im} \varphi^{\prime} \longrightarrow \mathfrak{m}^{\prime}$ be sections of $\pi^{\prime}$ and $\varphi^{\prime}$. Since $\pi^{\prime}(G s)=\pi(s)=I d$, we get another section $G s=\left(G_{1} s_{1}, G_{0} s_{0}\right)$ of $\pi^{\prime}$. Taking into account Lemma 5.12, we choose $s^{\prime}=G s$. Set

$$
\left\{\begin{aligned}
B_{0}(a) & =\left(F_{0} q_{0}-q_{0}^{\prime} G_{0}\right)\left(d s_{1}(a)-s_{0} d(a)\right) \\
B_{1}(x, y) & =\left(F_{0} q_{0}-q_{0}^{\prime} G_{0}\right)\left(\left[s_{0}(x), s_{0}(y)\right]-s_{0}[x, y]\right) \\
B_{2}(x, a) & =\left(F_{1} q_{1}-q_{1}^{\prime} G_{1}\right)\left(\left[s_{0}(x), s_{1}(a)\right]-s_{1}[x, a]\right) \\
B_{3}(x, y, z) & =\left(F_{1} q_{1}-q_{1}^{\prime} G_{1}\right)\left(l_{3}\left(s_{0}(x), s_{0}(y), s_{0}(z)\right)-s_{1} l_{3}(x, y, z)\right) .
\end{aligned}\right.
$$

Noticing that $\varphi^{\prime} F=G \varphi$, it is obvious that $B=\sum_{i=0}^{3} B_{i} \in C^{2}(\mathfrak{h}, \mathbb{V})$. Furthermore, relying on the properties of $(F, G)$, we obtain

$$
\begin{aligned}
& \left(\theta_{\varepsilon 0}-\theta_{\varepsilon^{\prime} 0}\right)(x, a) \\
= & F_{0}\left(\theta_{\varepsilon 0}(x, a)\right)-\theta_{\varepsilon^{\prime} 0}(x, a) \\
= & F_{0}\left(s_{0}(x) \triangleright q_{0}\left(d s_{1}(a)-s_{0}(d a)\right)-q_{0}\left(d s_{1}[x, a]-s_{0} d[x, a]\right)\right. \\
& \left.+q_{0}\left(\left[s_{0}(x), s_{0}(d a)\right]-s_{0}[x, d a]\right)-d^{\mathfrak{m}} q_{1}\left(\left[s_{0}(x), s_{1}(a)\right]-s_{1}[x, a]\right)\right) \\
& -G_{0} s_{0}(x) \triangleright^{\prime} q_{0}^{\prime}\left(d^{\prime} G_{1} s_{1}(a)-G_{0} s_{0}(d a)\right)+q_{0}^{\prime}\left(d^{\prime} G_{1} s_{1}[x, a]-G_{0} s_{0} d[x, a]\right) \\
& -q_{0}^{\prime}\left(\left[G_{0} s_{0}(x), G_{0} s_{0}(d a)\right]-G_{0} s_{0}[x, d a]\right)+d^{\mathfrak{m}^{\prime}} q_{1}^{\prime}\left(\left[G_{0} s_{0}(x), G_{1} s_{1}(a)\right]-G_{1} s_{1}[x, a]\right) \\
= & G_{0} s_{0}(x) \triangleright^{\prime}\left(F_{0} q_{0}-q_{0}^{\prime} G_{0}\right)\left(d s_{1}(a)-s_{0}(d a)\right)-\left(F_{0} q_{0}-q_{0}^{\prime} G_{0}\right)\left(d s_{1}[x, a]-s_{0} d[x, a]\right) \\
& \left.+\left(F_{0} q_{0}-q_{0}^{\prime} G_{0}\right)\left(\left[s_{0}(x), s_{0}(d a)\right]-s_{0}[x, d a]\right)-d^{\mathfrak{m}^{\prime}}\left(F_{1} q_{1}-q_{1}^{\prime} G_{1}\right)\left(\left[s_{0}(x), s_{1}(a)\right]-s_{1}[x, a]\right)\right) \\
= & \left(d_{\phi^{\prime}}^{(1,0)} B_{0}+\hat{d} B_{1}+\widehat{d^{\mathfrak{m}^{\prime}}} B_{2}\right)(x, a) .
\end{aligned}
$$

Similarly, considering the fact that $\phi$ and $\phi^{\prime}$ induce the same $\mathfrak{h}$-module structure on $\mathbb{V}$, we get

$$
\theta_{\varepsilon}-\theta_{\varepsilon^{\prime}}=D^{\mathfrak{h}} B .
$$

This finishes the proof.

Step 3: We show $\mu$ is a surjection, which follows from Corollary 5.4 and the following lemma.

Lemma 5.14. For any $[\theta] \in \mathrm{H}^{3}(\mathfrak{h}, \mathbb{V})$, we have $\mu\left(\mathcal{C}(\mathfrak{h}, \mathbb{V})_{[\theta]}\right)=[\theta]$.

Proof. For a crossed module $\varepsilon_{\lambda}=\left(\operatorname{ker} \pi \oplus_{\lambda} \mathbb{V}, \mathfrak{F}(\mathfrak{h}), \phi^{\lambda}, \varphi\right) \in \mathcal{C}(\mathfrak{h}, \mathbb{V})_{[\theta]}$, consider the complex

$$
\varepsilon_{\lambda}: 0 \rightarrow \mathbb{V} \stackrel{i}{\longrightarrow} \operatorname{ker} \pi \oplus_{\lambda} \mathbb{V} \stackrel{\varphi}{\longrightarrow} \mathfrak{F}(\mathfrak{h}) \stackrel{\pi}{\longrightarrow} \mathfrak{h} \rightarrow 0 .
$$

Choosing any section $s$ of $\pi$ and defining section $q$ of $\varphi$ on $\operatorname{Im} \varphi$ by $q(\alpha)=(\alpha, 0), \forall \alpha \in \operatorname{ker} \pi$, we get a 3-cocycle $\theta_{\varepsilon_{\lambda}} \in C^{3}(\mathfrak{h}, \mathbb{V})$. We claim that $\theta_{\varepsilon_{\lambda}}+\mathrm{D}^{\mathfrak{h}} s^{*} \lambda=\theta$, which implies that $\mu\left(\varepsilon_{\lambda}\right)=\left[\theta_{\varepsilon_{\lambda}}\right]=[\theta]$. Actually, by (33) and (10), we have

$$
\begin{aligned}
\left(\theta_{\varepsilon_{\lambda}}\right)_{0}(x, a)= & s_{0} x \triangleright_{\lambda}\left(d s_{1} a-s_{0} d a\right)-d s_{1}[x, a]+s_{0} d[x, a] \\
& +\left[s_{0} x, s_{0} d a\right]-s_{0}[x, d a]-d^{\lambda}\left(\left[s_{0} x, s_{1} a\right]-s_{1}[x, a]\right) \\
= & \lambda_{1}\left(s_{0} x, d s_{1} a-s_{0} d a\right)-\lambda_{0}\left(\left[s_{0} x, s_{1} a\right]-s_{1}[x, a]\right),
\end{aligned}
$$

and

$$
\left(D^{\mathfrak{h}} s^{*} \lambda\right)_{0}(x, a)=x \triangleright \lambda_{0}\left(s_{1} a\right)-\lambda_{0} s_{1}[x, a]+\lambda_{1}\left(s_{0} x, s_{0} d a\right)-d^{\mathbb{V}} \lambda_{2}\left(s_{0} x, s_{1} a\right) .
$$


Adding them together, we get

$$
\begin{aligned}
\left(\theta_{\varepsilon_{\lambda}}+\mathrm{D}^{\mathfrak{h}} s^{*} \lambda\right)_{0}(x, a) & =x \triangleright \lambda_{0}\left(s_{1} a\right)-\lambda_{0}\left[s_{0} x, s_{1} a\right]+\lambda_{1}\left(s_{0} x, d s_{1} a\right)-d^{\mathbb{V}} \lambda_{2}\left(s_{0} x, s_{1} a\right) \\
& =\left(D^{\mathfrak{F}(\mathfrak{h})} \lambda\right)_{0}\left(s_{0} x, s_{1} a\right)=\left(\pi^{*} \theta\right)_{0}\left(s_{0} x, s_{1} a\right) \\
& =\theta_{0}(x, a) .
\end{aligned}
$$

Likewise, we can obtain $\theta_{\varepsilon_{\lambda}}+\mathrm{D}^{\mathfrak{h}} s^{*} \lambda=\theta$. This finishes the proof.

Step 4: We shall prove for two crossed modules $\varepsilon, \varepsilon^{\prime} \in \mathcal{C}(\mathfrak{h}, \mathbb{V}), \mu(\varepsilon)=\mu\left(\varepsilon^{\prime}\right)$ iff $\varepsilon \sim \varepsilon^{\prime}$. Then, the map $\mu: \mathcal{C}(\mathfrak{h}, \mathbb{V}) \longrightarrow \mathrm{H}^{3}(\mathfrak{h}, \mathbb{V})$ induces a bijection between $\mathcal{C}(\mathfrak{h}, \mathbb{V}) / \sim$ and $\mathrm{H}^{3}(\mathfrak{h}, \mathbb{V})$.

A direct consequence of Lemma 5.13 and 5.14 is:

Corollary 5.15. If $\varepsilon \sim \varepsilon^{\prime}$, we have $\mu(\varepsilon)=\mu\left(\varepsilon^{\prime}\right)$.

Proposition 5.16. For a 3-cocycle $\theta \in C^{3}(\mathfrak{h}, \mathbb{V}), \mu(\varepsilon)=[\theta]$ if and only if there exists a crossed module $\varepsilon_{\lambda} \in \mathcal{C}(\mathfrak{h}, \mathbb{V})_{[\theta]}$ and a strong map $(F, G): \varepsilon_{\lambda} \longrightarrow \varepsilon$. That is, if $\mu(\varepsilon)=\mu\left(\varepsilon^{\prime}\right)$, then $\varepsilon \sim \varepsilon^{\prime}$.

Proof. For such a crossed module $\varepsilon=(\mathfrak{m}, \mathfrak{g}, \phi, \varphi) \in \mathcal{C}(\mathfrak{h}, \mathbb{V})$, choosing sections $s: \mathfrak{h} \longrightarrow \mathfrak{g}$ and $q: \operatorname{Im} \varphi \longrightarrow \mathfrak{m}$ of $\pi^{\prime}$ and $\varphi$ respectively, we can construct $\lambda_{\varepsilon}$ and then a 3-cocycle $\theta_{\varepsilon} \in C^{3}(\mathfrak{h}, \mathbb{V})$ as in (33) such that $\left[\theta_{\varepsilon}\right]=[\theta]$. Let $G: \mathfrak{F}(\mathfrak{h}) \longrightarrow \mathfrak{g}$ be the strong Lie 2-algebra homomorphism induced by $s$ (the property of free Lie 2-algebras). Define linear maps $\psi=\left(\psi_{0}, \psi_{1}\right): \mathfrak{F}(\mathfrak{h}) \longrightarrow \mathfrak{m}$ by $\psi(\bar{x})=q(G \bar{x}-s \pi \bar{x}), \forall \bar{x} \in \mathfrak{F}(\mathfrak{h})$. Next, construct four maps:

$$
\left\{\begin{aligned}
\lambda_{0}(\bar{a})= & \lambda_{\varepsilon 0}\left(\pi_{1} \bar{a}\right)+d^{\mathfrak{m}} \psi_{1} \bar{a}-\psi_{0} d \bar{a}, \\
\lambda_{1}(\bar{x}, \bar{y})= & \lambda_{\varepsilon 1}\left(\pi_{0} \bar{x}, \pi_{0} \bar{y}\right)-\psi_{0}[\bar{x}, \bar{y}]-\left[\psi_{0} \bar{x}, \psi_{0} \bar{y}\right]+G_{0} \bar{x} \triangleright \psi_{0} \bar{y}-G_{0} \bar{y} \triangleright \psi_{0} \bar{x}, \\
\lambda_{2}(\bar{x}, \bar{a})= & \lambda_{\varepsilon 2}\left(\pi_{1} \bar{x}, \pi_{1} \bar{a}\right)-\psi_{1}[\bar{x}, \bar{a}]-\left[\psi_{0} \bar{x}, \psi_{1} \bar{a}\right]+G_{0} \bar{x} \triangleright \psi_{1} \bar{a}-G_{1} \bar{a} \triangleright \psi_{0} \bar{x}, \\
\lambda_{3}(\bar{x}, \bar{y}, \bar{z})= & \lambda_{\varepsilon 3}\left(\pi_{0} \bar{x}, \pi_{0} \bar{y}, \pi_{0} \bar{z}\right)-\psi_{1} l_{3}(\bar{x}, \bar{y}, \bar{z})+l_{3}^{\mathfrak{m}}\left(\psi_{0} \bar{x}, \psi_{0} \bar{y}, \psi_{0} \bar{z}\right) \\
& -\left(l_{\phi_{0}(G \bar{x})}\left(\psi_{0} \bar{y}, \psi_{0} \bar{z}\right)+\left(G_{0} \bar{x}, G_{0} \bar{y}\right) \triangleright \psi_{0} \bar{z}+\text { c.p. }\right),
\end{aligned}\right.
$$

for any $\bar{x}, \bar{y}, \bar{z} \in \mathfrak{F}(\mathfrak{h})_{0}, \bar{a} \in \mathfrak{F}(\mathfrak{h})_{1}$. Since $\pi, G$ and $\varphi$ commute with $d$, we have

$$
\begin{aligned}
\varphi_{0}\left(\lambda_{0}(\bar{a})\right) & =d s_{1} \pi_{1} \bar{a}-s_{0} d \pi_{1} \bar{a}+d\left(G_{1} \bar{a}-s_{1} \pi_{1} \bar{a}\right)-\left(G_{0} d \bar{a}-s_{0} \pi_{0} d \bar{a}\right) \\
& =0 .
\end{aligned}
$$

Similarly, we can get $\varphi \circ \lambda_{i}=0, i=1,2,3$, that is, $\lambda=\sum_{i=0}^{3} \lambda_{i} \in C^{2}(\mathfrak{F}(\mathfrak{h}), \mathbb{V})$.

Moreover, we claim that $\mathrm{D}^{\mathfrak{F}(\mathfrak{h})} \lambda=\pi^{*} \theta_{\varepsilon}$. By straightforward calculations, we get

$$
\begin{aligned}
\left(\mathrm{D}^{\mathfrak{F}(\mathfrak{h})} \lambda\right)_{0}(\bar{x}, \bar{a})= & \pi_{0} \bar{x} \triangleright \lambda_{0}(\bar{a})-\lambda_{0}[\bar{x}, \bar{a}]+\lambda_{1}(\bar{x}, d \bar{a})-d^{\mathfrak{m}} \lambda_{2}(\bar{x}, \bar{a}) \\
= & \pi_{0} \bar{x} \triangleright\left(\lambda_{\varepsilon 0}\left(\pi_{1} \bar{a}\right)+d^{\mathfrak{m}} \psi_{1} \bar{a}-\psi_{0} d \bar{a}\right)-\lambda_{\varepsilon 0}\left(\pi_{1}[\bar{x}, \bar{a}]\right)-d^{\mathfrak{m}} \psi_{1}[\bar{x}, \bar{a}]+\psi_{0} d[\bar{x}, \bar{a}] \\
& +\lambda_{\varepsilon 1}\left(\pi_{0} \bar{x}, \pi_{0} d \bar{a}\right)-\psi_{0}[\bar{x}, d \bar{a}]-\left[\psi_{0} \bar{x}, \psi_{0} d \bar{a}\right]+G_{0} \bar{x} \triangleright \psi_{0} d \bar{a}-G_{0} d \bar{a} \triangleright \psi_{0} \bar{x} \\
& -d^{\mathfrak{m}}\left(\lambda_{\varepsilon 2}\left(\pi_{0} \bar{x}, \pi_{1} \bar{a}\right)-\psi_{1}[\bar{x}, \bar{a}]-\left[\psi_{0} \bar{x}, \psi_{1} \bar{a}\right]+G_{0} \bar{x} \triangleright \psi_{1} \bar{a}-G_{1} \bar{a} \triangleright \psi_{0} \bar{x}\right) \\
= & s_{0} \pi_{0} \bar{x} \triangleright \lambda_{\varepsilon 0}\left(\pi_{1} \bar{a}\right)-\lambda_{\varepsilon 0}\left(\pi_{1}[\bar{x}, \bar{a}]\right)+\lambda_{\varepsilon 1}\left(\pi_{0} \bar{x}, \pi_{0} d \bar{a}\right)-d^{\mathfrak{m}} \lambda_{\varepsilon 2}\left(\pi_{0} \bar{x}, \pi_{1} \bar{a}\right) \\
= & \theta_{\varepsilon 0}\left(\pi_{0} \bar{x}, \pi_{1} \bar{a}\right) \\
= & \left(\pi^{*} \theta_{\varepsilon 0}\right)(\bar{x}, \bar{a}),
\end{aligned}
$$

where we have used condition $(i)$ of Definition 3.2 , Likewise, we deduce that $\mathrm{D}^{\mathfrak{F}(\mathfrak{h})} \lambda=\pi^{*} \theta_{\varepsilon}$.

So we can use such a defined $\lambda$ to construct a crossed module $\varepsilon_{\lambda}$ as in Proposition 5.2 . In the following, we prove there is a map $(F, G)$ from $\varepsilon_{\lambda}$ to $\varepsilon$ in $\mathcal{C}(\mathfrak{h}, \mathbb{V})$ :

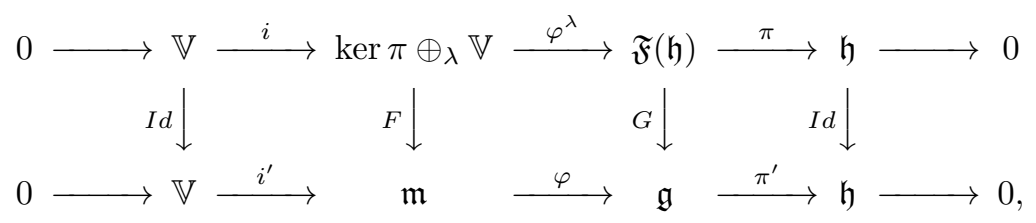


where $F(\alpha+u)=i^{\prime}(u)+q G(\alpha), \forall \alpha \in \operatorname{ker} \pi, u \in \mathbb{V}$. Indeed, we shall prove $F$ is a strong Lie 2-algebra homomorphism compatible with the actions, that is, for any $\alpha \in \operatorname{ker} \pi, u \in \mathbb{V}, \bar{x} \in \mathfrak{F}(\mathfrak{h})$,

$$
F\left(\bar{x} \triangleright_{\lambda}(\alpha+u)\right)=G(\bar{x}) \triangleright F(\alpha+u), \quad F\left((\bar{x}, \bar{y}) \triangleright_{\lambda}(\alpha+u)\right)=(G \bar{x}, G \bar{y}) \triangleright F(\alpha+u) .
$$

We only sketch the proof of compatibility. For any $\alpha \in \operatorname{ker} \pi_{0}, u \in V_{0}$ and $\bar{x}_{0} \in \mathfrak{F}(\mathfrak{h})_{0}$, we have

$$
\begin{aligned}
F_{0}\left(\bar{x} \triangleright_{\lambda}(\alpha+u)\right)= & F_{0}\left([\bar{x}, \alpha]+\lambda_{1}(\bar{x}, \alpha)+\pi_{0} \bar{x} \triangleright u\right) \\
= & \lambda_{1}(\bar{x}, \alpha)+\pi_{0} \bar{x} \triangleright i_{0}^{\prime} u+q_{0} G_{0}[\bar{x}, \alpha] \\
= & -q_{0} G_{0}[\bar{x}, \alpha]-\left[q_{0} G_{0} \bar{x}-q_{0} s_{0} \pi_{0} \bar{x}, q_{0} G_{0} \alpha\right]+G_{0} \bar{x} \triangleright q_{0} G_{0} \alpha \\
& -G_{0} \alpha \triangleright\left(q_{0} G_{0} \bar{x}-q_{0} s_{0} \pi_{0} \bar{x}\right)+\pi_{0} \bar{x} \triangleright i_{0}^{\prime} u+q_{0} G_{0}[\bar{x}, \alpha] \\
= & \pi_{0} \bar{x} \triangleright i_{0}^{\prime} u+G_{0} \bar{x} \triangleright q_{0} G_{0} \alpha \\
= & G_{0} \bar{x} \triangleright F_{0}(\alpha+u),
\end{aligned}
$$

where we have used the condition $(i)$ of Definition 3.2 and $\pi_{0} \bar{x} \triangleright i_{0}^{\prime} u=\pi_{0}^{\prime} G_{0} \bar{x} \triangleright i_{0}^{\prime} u=G_{0} \bar{x} \triangleright i_{0}^{\prime} u$. Next, since $\left(\pi_{0} \bar{x}, \pi_{0} \bar{y}\right) \triangleright i_{0}^{\prime} u=\left(G_{0} \bar{x}, G_{0} \bar{y}\right) \triangleright i_{0}^{\prime} u$ due to the fixed action of $\mathfrak{h}$ on $\mathbb{V}$, we have

$$
\begin{aligned}
& F_{1}\left((\bar{x}, \bar{y}) \triangleright_{\lambda}(\alpha+u)\right) \\
= & F_{1}\left(l_{3}(\bar{x}, \bar{y}, \alpha)+\lambda_{3}(\bar{x}, \bar{y}, \alpha)+\left(\pi_{0} \bar{x}, \pi_{0} \bar{y}\right) \triangleright u\right) \\
= & -q_{1} G_{1} l_{3}(\bar{x}, \bar{y}, \alpha)+l_{3}^{\mathfrak{m}}\left(q_{0} G_{0} \bar{x}-q_{0} s_{0} \pi_{0} \bar{x}, q_{0} G_{0} \bar{y}-q_{0} s_{0} \pi_{0} \bar{y}, q_{0} G_{0} \alpha\right) \\
& -\left(G_{0} \bar{x}, G_{0} \bar{y}\right) \triangleright q_{0} G_{0} \alpha-\left(G_{0} \bar{y}, G_{0} \alpha\right) \triangleright\left(q_{0} G_{0} \bar{x}-q_{0} s_{0} \pi_{0} \bar{x}\right)-\left(G_{0} z, G_{0} \bar{x}\right) \triangleright\left(q_{0} G_{0} \bar{y}-q_{0} s_{0} \pi_{0} \bar{y}\right) \\
& +\left(G_{0} \bar{x}, G_{0} \bar{y}-s_{0} \pi_{0} \bar{y}\right) \triangleright q_{0} G_{0} \alpha+\left(G_{0} \bar{y}, G_{0} \alpha\right) \triangleright\left(q_{0} G_{0} \bar{x}-q_{0} s_{0} \pi_{0} \bar{x}\right) \\
& +\left(G_{0} \alpha, G_{0} \bar{x}-s_{0} \pi_{0} \bar{x}\right) \triangleright\left(q_{0} G_{0} \bar{y}-q_{0} s_{0} \pi_{0} \bar{y}\right)+q_{1} G_{1} l_{3}(\bar{x}, \bar{y}, \alpha)+\left(\pi_{0} \bar{x}, \pi_{0} \bar{y}\right) \triangleright i_{0}^{\prime} u \\
= & \left(\pi_{0} \bar{x}, \pi_{0} \bar{y}\right) \triangleright i_{0}^{\prime} u-\left(G_{0} \bar{x}, G_{0} \bar{y}\right) \triangleright q_{0} G_{0} \alpha \\
= & \left(G_{0} \bar{x}, G_{0} \bar{y}\right) \triangleright F_{0}(\alpha+u),
\end{aligned}
$$

where we have used condition $(i),(i i),(i i i)$ of Definition 3.2 The other hand is a consequence of Lemma 5.13 and 5.14. This ends the proof.

Next, we give an alternative description of the equivalence relation in Theorem 5.10, which is similar to the statements in 24, 4. Two strong crossed modules $(\mathfrak{m}, \mathfrak{g}, \phi, \varphi),\left(\mathfrak{m}^{\prime}, \mathfrak{g}^{\prime}, \phi^{\prime}, \varphi^{\prime}\right) \in \mathcal{C}(\mathfrak{h}, \mathbb{V})$ are called elementary equivalent if there is a morphism of crossed modules $(F, G, \tau)$ such that the diagram

is commutative and

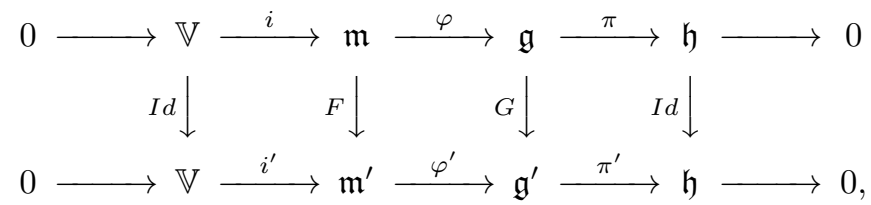

$$
G_{2}=0 ; \quad \operatorname{Im} \tau \subset i^{\prime}(\mathbb{V}) ; \quad \tau\left(\mathfrak{g}_{0} \wedge i(\mathbb{V})\right)=0 ; \quad \tau\left(\varphi_{0} \alpha, \beta\right)=\tau\left(\alpha, \varphi_{0} \beta\right), \quad \forall \alpha, \beta \in \mathfrak{m}_{0} .
$$

By a straightforward but tedious computation combined with the proof of Theorem 5.10, we obtain the following proposition.

Proposition 5.17. The equivalence relation generated by elementary equivalence relation coincides with the equivalence relation $\sim$ in Theorem 5.10 .

One key step in the proof of Theorem 5.10 is the construction of the map $\mu: \mathcal{C}(\mathfrak{h}, \mathbb{V}) \rightarrow \mathrm{H}^{3}(\mathfrak{h}, \mathbb{V})$. The following example illustrates that for a particular class of crossed modules given in Example 3.10. the map $\mu$ is closely related to the connecting map in the long exact sequence of cohomology groups. 
Example 5.18. Consider the strong crossed module $\varepsilon=\left(\mathbb{I}, \mathfrak{h} \oplus_{\lambda} \mathbb{Q}, \phi, \varphi\right) \in \mathcal{C}(\mathfrak{h}, \mathbb{V})$ obtained in Example 3.10. The sequence (15) induces a short sequence of complexes

$$
0 \rightarrow C^{*}(\mathfrak{h}, \mathbb{V}) \stackrel{\bar{p}}{\longrightarrow} C^{*}(\mathfrak{h}, \mathbb{I}) \stackrel{\bar{q}}{\longrightarrow} C^{*}(\mathfrak{h}, \mathbb{Q}) \rightarrow 0 .
$$

Since for arbitrary $\mathfrak{h}$-module homomorphism $f$, we have $\bar{f} \circ D^{\mathfrak{h}}=D^{\mathfrak{h}} \circ \bar{f}$. Thus $p, q$ induce maps between cohomology groups. Moreover, similar to the process in homological algebra, we can construct a connecting homomorphism $\partial: \mathrm{H}^{*}(\mathfrak{h}, \mathbb{Q}) \rightarrow \mathrm{H}^{*+1}(\mathfrak{h}, \mathbb{V})$ such that

$$
\cdots \rightarrow \mathrm{H}^{*}(\mathfrak{h}, \mathbb{V}) \stackrel{\bar{p}}{\longrightarrow} \mathrm{H}^{*}(\mathfrak{h}, \mathbb{I}) \stackrel{\bar{q}}{\longrightarrow} \mathrm{H}^{*}(\mathfrak{h}, \mathbb{Q}) \stackrel{\partial}{\longrightarrow} \mathrm{H}^{*+1}(\mathfrak{h}, \mathbb{V}) \rightarrow \cdots
$$

is a long exact sequence of cohomology groups.

In particular, we have $\mu(\varepsilon)=\partial[\lambda]$. See [24, Theorem 3] for more details.

\section{References}

[1] J.C. Baez and A.S. Crans, Higher-dimensional algebra VI: Lie 2-algebras, Theory Appl. Categ., 2004, 12: 492-538.

[2] C.M. Bai, Y.H. Sheng and C.C. Zhu, Lie 2-bialgebra, Comm. Math. Phys, 2013, 320(1): 149-172.

[3] H.J. Baues and E.G. Minian, Crossed extensions of algebras and Hochschild cohomology, Homol. Homot. Appl., 2002, 4(2): 63-82.

[4] J.M. Casas, E. Khmaladze and M. Ladra, Crossed modules for Leibniz n-algebras, Forum Math., 2008, 20(5): 841-858.

[5] S. Chen, Y. Sheng and Z. Zheng, Non-abelian extensions of Lie 2-algebras, Sci. China Math., 2012, 55(8): 1655-1668.

[6] Z. Chen, M. Stinon and P. Xu, Weak Lie 2-bialgebras, J. Geom. Phy., 2013, 68: 59-68.

[7] D. Conduché, Modules croises generalises de Longueur 2, J. Pure Appl. Alg. 1984, 34: 155178.

[8] G.J. Ellis, Homotopical aspects of Lie algebras, J. Austral. Math. Soc., Ser. A, 1993, 54(3): 393-419.

[9] J. Faria Martins and R. Picken, The fundamental Gray 3-groupoid of a smooth manifold and local 3-dimensional holonomy based on a 2-crossed module, Diff. Geom. Appl., 2011, 29(2): 179-206.

[10] M. Gerstenhaber, The cohomology structure of an associate ring, Ann. Math., 1963, 78(2): 267-288.

[11] M. Gerstenhaber, A uniform cohomology theory for algebras, Pros. Nat. Acad. Sci. U.S.A., 1964, 51: 626-629.

[12] G. Ginot and P. Xu, Cohomology of Lie 2-groups, LEnseignement Mathmatique, 2009, 55(3): 373-396.

[13] J.L. Loday, Spaces having finitely many non-trivial homotopy groups, J. Pure Appl. Alg, 1982, 24: 179-202. 
[14] T. Lada and M. Markl, Strongly homotopy Lie algebras, Comm. Alg. 1995, 23(6): 2147-2161.

[15] Z.J. Liu, Y.H. Sheng and T. Zhang, Deformations of Lie 2-algebras, arXiv: 1306.6225.

[16] M. Markl, A cohomology theory for A (m)-algebras and applications, J. Pure Appl. Alg., 1992, 83(2): 141-175.

[17] M. Markl, Free homotopy algebras, Homol. Homot. Appl., 2005, 7(2): 123-137.

[18] J. Millès, André-Quillen cohomology of algebras over an operad, Adv. Math., 2011, 226(6): $5120-5164$.

[19] I. Moerdijk, Orbifolds as groupoids: an introduction, Orbifolds in mathematics and physics (Madison, WI, 2001), Contemp. Math., 2002, 310, $205 \mathrm{C} 222$.

[20] A. Mutlu and T. Porter, Crossed squares and 2-crossed modules, arXiv: 0210462.

[21] K.L. Norrie, Actions and automorphisms of crossed modules, Bull. Soc. Math. France, 1990, 118(2): 129-146.

[22] M. Penkava, L-infinity algebras and their cohomology, arXiv: 9512014.

[23] M. Schlessinger and J. Stasheff, The Lie algebra structure of tangent cohomology and deformation theory, J. Pure Appl. Alg. 1985, 38: 313-322.

[24] F. Wagemann, On Lie algebra crossed modules, Comm. Alg., 2006, 34(5): 1699-1722. 